submitted papers

\title{
Polarization-Sensitive CARS of Excited-State Rhodamine 6G: Induced Anisotropy Effects on Depolarization Ratios
}

\author{
GERALD W. LUCASSEN, WIM P. de BOEIJ, and JAN GREVE* \\ Applied Optics Group, Department of Applied Physics, University of Twente, P.O. Box 217, 7500 AE Enschede, The Netherlands
}

\begin{abstract}
Resonance polarization-sensitive coherent anti-Stokes Raman scattering (PS CARS) spectra of the electronic ground state and excited singlet $S_{1}$ state of rhodamine $6 \mathrm{G}$ in ethanol were obtained with the use of the pumpprobe technique with nanosecond time resolution. Variation of the polarization orientation of the pump laser beam showed differences in the excited-state spectra due to optically induced anisotropy. The pure electronic susceptibility of ground-state rhodamine $6 \mathrm{G}$ was shown to be small in comparison with nonresonant susceptibility of the solvent, and was neglected in further analyses. The pure electronic susceptibility of excited rhodamine 6G was examined by coherent ellipsometry. The complex third-order susceptibility was analyzed by means of a nonlinear least-squares fit program that provides detailed information on the Raman vibration parameters, including depolarization ratios and phases. In the isotropic case the measured depolarization ratios are close to $1 / 3$, whereas in the anisotropic case, ground-state depolarization ratios are $0.5-0.65$ and in the excited state $0.17-0.22$. Estimated depolarization ratio changes in ground-state and excited-state rhodamine 6G are in agreement with theoretically predicted values in the case of induced anisotropy under the assumption of parallel dipole moments of the CARS process. The effects of possible changed molecular structure or symmetry and changed enhancement of different electronic transitions cannot be determined without making some assumptions about one of these effects. The obtained phase differences reflect different enhancements and vibronic coupling for ground-state and excited-state vibrations. The groundstate and excited-state hyperpolarizabilities, $\gamma_{s_{0}}^{\mathrm{E}}$ and $\gamma_{s_{1}}^{\mathrm{E}}$, of rhodamine 6G were estimated to be $3.8 \cdot 10^{-35}$ esu and $27.4 \cdot 10^{-35}$ esu, respectively. Index Headings: Nonlinear spectroscopy; Raman spectroscopy; Excitedstate spectroscopy; Coherent ellipsometry.
\end{abstract}

\section{INTRODUCTION}

Coherent anti-Stokes Raman scattering (CARS) offers the possibility of measuring vibrational spectra of molecules with high signal efficiency, as is well known. ${ }^{1}$ Due to its insensitivity to fluorescence, CARS allows measurements in the electronic excited states, which often are inaccessible to spontaneous Raman scattering (RS). Besides the usual spectroscopic identification of the molecules by vibrational frequencies, bandwidths, and amplitudes, the depolarization ratios, containing information on symmetry and structure of the molecules, can be obtained by use of the polarization-sensitive CARS (PS CARS) technique. ${ }^{2}$ In addition, the coherent Raman

\footnotetext{
Received 20 January 1993; revision received 13 April 1993.

* Author to whom correspondence should be sent.
}

technique (as its name implies) renders determination of vibrational phases relative to that of the nonresonant background, although the phase is not often used as a parameter. These phases contain information on the influence of vibronic coupling and enhancement mechanisms of different electronic transitions.

In the last few years, application of (time-resolved) RS and CARS to the study of molecules in excited electronic states has grown rapidly. ${ }^{3-8}$ For example, studies on the photo-isomerization processes of trans-stilbene $e^{4,5}$ and dye molecules ${ }^{6,7}$ and the short-lived excited states of polyatomic molecules ${ }^{8}$ by using pump-probe techniques have proven to be very informative. Polarization-sensitive RS measurements by Hamaguchi et al., ${ }^{9}$ on $S_{1}$-excited trans stilbene when pumped with UV light, yielded depolarization ratios that were unusually high compared with that of the unpumped (ground-state) molecules. These differences probably indicate a changed molecular symmetry in the excited state, Kamalov et al. ${ }^{5}$ used picosecond time-resolved PS CARS and coherent anti-Stokes Raman ellipsometry (CARE) to study $S_{1}$-excited transstilbene in solution. It was shown that partially ordered excited molecules have considerably different electronic third-order susceptibilities under linearly polarized UV excitation in comparison with those of isotropic media.

With respect to the estimation of vibrational depolarization ratios under resonant excitation (pumping) in anisotropic media in the excited state (and in the ground state) with the use of CARS, less information is available in the literature. Therefore, we have carried out a detailed PS CARS study of excited-state rhodamine 6G using the pump-CARS probe technique with nanosecond time resolution. Rhodamine $6 \mathrm{G}$ in ethanol solution was chosen as a test object for two reasons: First, its fluorescence lifetime $\tau_{f 1}$ is $5 \mathrm{~ns},{ }^{10}$ which easily leads to a high degree of excited molecules when excited in the lowest singlet $S_{1}$ electronic absorption band with the use of 8-ns pulses. Second, since rhodamine $6 \mathrm{G}$ has been studied extensively, ${ }^{11,12}$ the electronic structure and orientation of the transition moments of the lowest electronic transitions have been determined. Band positions of the ground and $S_{1}$ excited state are known from resonance CARS measurements ${ }^{3,7,13}$ previously applied to rhodamine $6 \mathrm{G}$ in solutions, which simplifies the analysis. With induced anisotropy in the molecular orientation distri- 
bution by photoselection with a linearly polarized pump beam or by partial ordering of the molecules in the strong pump field, polarization characteristics are expected to be different from the unpumped (isotropic) case.

The aim of this work is to show that different aspects (viz., optically induced anisotropy, molecular symmetry, and electronic enhancement mechanisms) influence the depolarization ratios in the electronic ground and excited state upon pumping as measured by PS CARS. Here we use a combination of techniques, measurements (absorption, concentration variation, PS CARS, coherent ellipsometry), and fitting procedures which, in our view, are necessary to determine vibrational depolarization ratios and molecular hyperpolarizabilities in both the ground state and singlet excited state.

New aspects concern (1) the determination of depolarization ratios of ground-state $\left(S_{0}\right)$ and excited-state $\left(S_{1}\right)$ rhodamine $6 \mathrm{G}$ by measurement of the $\chi_{1111}^{(3)}$ and $\chi_{1221}^{(3)}\left(\right.$ or $\left.\chi_{212}^{(3)}\right)$ components of the vibrational third-order susceptibility and comparison with theoretical values; (2) investigations of the total electronic third-order susceptibility by means of concentration variations and coherent ellipsometry to distinguish between solvent, $S_{0}$-state rhodamine $6 \mathrm{G}$, and $S_{1}$-state rhodamine $6 \mathrm{G}$ contributions; (3) determination of vibrational parameters of both $S_{0}$ and $S_{1}$-state rhodamine $6 \mathrm{G}$ by spectral analyses of a set of PS CARS spectra with the use of a nonlinear leastsquares fit program; and (4) determination of $S_{1}$-state hyperpolarizability.

The second section of this paper contains a short description of polarization-sensitive CARS theory; experimental aspects are given in the third section, followed by the results, discussion, and conclusions. Some mathematical derivations of susceptibility tensors in anisotropic systems and coherent ellipsometry analyses are given in Appendices A and B, respectively.

\section{PS CARS THEORY}

In single degenerate PS CARS, the detected signal intensity $I_{A}$ at the anti-Stokes frequency $\omega_{a}=2 \omega_{1}-\omega_{2}$ is given by ${ }^{1,2}$

$$
\begin{aligned}
I_{A}\left(\omega_{a}\right) \sim & \left|\left(\vec{e}_{A}^{*} \cdot \vec{P}^{(3)}\left(\omega_{a}\right)\right)\right|^{2} \\
\sim & \mid \vec{e}_{A}^{*} \cdot\left(\chi_{1111}^{(3)} \vec{e}_{1}\left(\vec{e}_{1} \cdot \vec{e}_{2}^{*}\right)\right. \\
& \left.\quad+\chi_{1221}^{(3)}\left(\vec{e}_{2}^{*}-\vec{e}_{1}\left(\vec{e}_{1} \cdot \vec{e}_{2}^{*}\right)\right)\right)\left.E_{1}^{2} E_{2}^{*}\right|^{2}
\end{aligned}
$$

where $\vec{e}_{A}^{*}, \vec{e}_{1}$, and $\vec{e}_{2}^{*}$ denote the unit polarization vectors of the analyzer and input laser fields, respectively. $\vec{P}^{(3)}$ is the induced total third-order polarization, and $\chi_{1111}^{(3)}$ and $\chi_{1221}^{(3)}$ are the polarization components of the cubic nonlinear susceptibility tensor (where $1,2=\mathrm{x}, \mathrm{y}$ ). $E_{1,2}$ are the amplitudes of the input electric fields. The asterisk (*) corresponds to an emitted photon. The total polarization $\vec{P}^{(3)}$ can be written as:

$$
\vec{P}^{(3)}=\vec{P}^{(3) \mathrm{NR}}+\vec{P}^{(3) \mathrm{E}}+\vec{P}^{(3) \mathrm{R}}
$$

where $\vec{P}^{(3) N R}$ denotes the nonvibration resonant electronic contribution which arises from the solvent. The last two terms on the right arise from the dissolved molecules. The pure resonant electronic polarization $\vec{P}^{(3) \mathrm{E}}$ is a nonvibration resonant contribution where any of the applied frequencies $\omega_{1}, \omega_{2}$, and $\omega_{a}$ may be close to electronic tran- sitions of the molecule. $P^{(3) \mathrm{R}}$ denotes the vibration resonant term and contains all Raman-active vibrations. In a similar way, the macroscopic (bulk) total third-order susceptibility $\chi^{(3)}$ is defined by

$$
\chi^{(3)}=\chi^{(3) \mathrm{NR}}+\chi^{(3) \mathrm{E}}+\chi^{(3) \mathrm{R}}
$$

where $\chi^{(3) \mathrm{NR}}, \chi^{(3) \mathrm{E}}$, and $\chi^{(3) \mathrm{R}}$ denote the nonresonant, pure electronic, and vibration resonant susceptibility, respectively. $\chi^{(3) \mathrm{NR}}$ is nearly frequency independent over the measured frequency range and can therefore be taken as a real constant. The pure electronic $\left(\chi^{(3) \mathrm{E}}\right)$ and vibration resonant $\left(\chi^{(3) R}\right)$ terms have complex values. Taking into account the electronic and vibrational resonances of solvent and dissolved molecules, Eq. 3 can be rewritten as:

$$
\begin{aligned}
& \chi^{(3)}(x)= N_{\mathrm{Sol}}\left(\gamma_{\mathrm{Sol}}^{(3) \mathrm{NR}}+\sum_{t} \frac{\gamma_{t S o l}^{(3) \mathrm{R}}}{\left(\Omega_{t \mathrm{Sol}}^{\mathrm{R}}-x\right) / \Gamma_{t \mathrm{Sol}}^{\mathrm{R}}-i}\right) \\
&+N_{0}\left(\sum _ { s } g _ { s } \left[\frac{\gamma_{s}^{(3) \mathrm{E}} \exp \left(i \theta_{s}^{\mathrm{E}}\right)}{\left(\Omega_{s}^{\mathrm{E}}-x\right) / \Gamma_{s}^{\mathrm{E}}-i}\right.\right. \\
&\left.\left.+\sum_{t} \frac{\gamma_{s t}^{(3) \mathrm{R}} \exp \left(i \theta_{s t}^{\mathrm{R}}\right)}{\left(\Omega_{s t}^{\mathrm{R}}-x\right) / \Gamma_{s t}^{\mathrm{R}}-i}\right]\right)
\end{aligned}
$$

where $x$ denotes the frequency difference $\left(\omega_{1}-\omega_{2}\right)$ which is the independent variable in the spectral scan. $\gamma_{\mathrm{Sol}}^{(3) \mathrm{NR}}, \gamma_{t \mathrm{Sol}}^{(3) \mathrm{R}}, \Omega_{t \mathrm{Sol}}^{\mathrm{R}}, \Gamma_{t \mathrm{Sol}}^{\mathrm{R}}$, and $N_{\mathrm{Sol}}$ denote nonresonant amplitude, vibration resonant amplitudes, frequencies, bandwidths, and concentration of the solvent molecules. $N_{0}$ is the concentration-dissolved molecules, and $g_{s}$ denotes the fraction of dissolved molecules in the electronic state $s$, where $\sum_{s} g_{s}=1 . \gamma_{s}^{(3) \mathrm{E}}, \theta_{s}^{\mathrm{E}}, \Omega_{s}^{\mathrm{E}}, \Gamma_{s}^{\mathrm{E}} \quad$ and $\gamma_{s t}^{(3) \mathrm{R}}, \theta_{s t}^{\mathrm{R}}, \Omega_{s t}^{\mathrm{R}}, \Gamma_{s t}^{\mathrm{R}}$ denote real amplitudes, phases, frequencies, and bandwidths of the electronic and vibration resonant transitions of the dissolved molecules in electronic state $s$, respectively. The summation (s) runs over all molecular electronic states including the ground state and the summation $(t)$ over all molecular vibrational states. In Eq. $4 \theta_{s}^{\mathrm{E}}$ and $\theta_{s t}^{\mathrm{R}}$ are relative phases with respect to that of the (real) nonresonant background, which is taken to be zero.

Equation 1 was derived for an isotropic medium. In the second part of the Results section, relations between the susceptibility components are derived for the anisotropic case.

Under the assumption of Kleinman symmetry (i.e., far away from electronic resonances) the relations between the nonresonant susceptibility components in the isotropic case are

$$
\chi_{1122}^{(3) \mathrm{NR}}=\chi_{1212}^{(3) \mathrm{NR}}=\chi_{1221}^{(3) \mathrm{NR}}=\frac{1}{3} \chi_{1111}^{(3) \mathrm{NR}} \text { or } \rho^{\mathrm{NR}}=\chi_{1221}^{(3)} / \chi_{1111}^{(3)}=\frac{1}{3}
$$

where $\rho^{\mathrm{NR}}$ is the nonresonant depolarization ratio. When one is applying polarizations $\vec{e}_{1}$ and $\vec{e}_{2}$ under an angle $\phi$, the different components $\chi_{1111}^{(3)}$ and $\chi_{1221}^{(3)}\left(=\chi_{2112}^{(3)}\right)$ can be measured by selecting different analyzer polarization orientations $\vec{e}_{A}$. Figure 1 shows two polarization schemes used to measure these components.

Vibration resonant polarizations $\vec{P}^{\mathrm{R}}$ with depolarization ratios $\rho^{\mathrm{R}}$ different from $\rho^{\mathrm{NR}}$ have different orienta- 


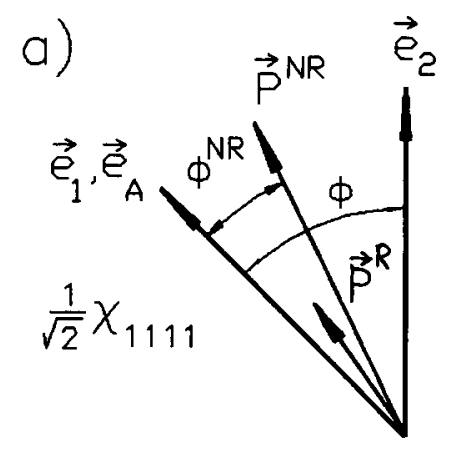

b)

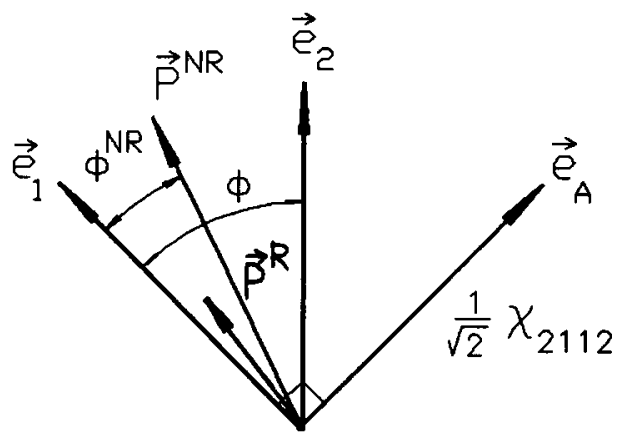

FIG. 1. Linear polarization conditions as set by Glan-Taylor prisms used to measure (a) $\chi_{1111}^{(3)}$ and (b) $\chi_{1221}^{(3)}$ or $\chi_{2112}^{(3)}$, with angle $\phi=45^{\circ}$ between the unit polarization vectors $\vec{e}_{1}$ and $\vec{e}_{2}$ of the CARS probe beams, and analyzer unit polarization vector $\vec{e}_{A}$. The nonresonant polarization $\vec{P}^{\mathrm{NR}}$ a resonant vector $\vec{P}^{\mathrm{R}}$, and their angles $\phi^{\mathrm{NR}}$ and $\theta^{\mathrm{R}}$ with respect to $\vec{e}_{1}$ are also depicted. tions with respect to $\vec{P}^{\mathrm{NR}}$. The angle $\theta^{\mathrm{R}}$ between $\vec{P}^{\mathrm{R}}$ and $\vec{e}_{1}$ is given by:

$$
\theta^{\mathrm{R}}=\arctan \left(\rho^{\mathrm{R}} \tan \phi\right)
$$

with $\rho^{\mathrm{R}}=\chi_{1221}^{(3)} / \chi_{1111}^{(3)}$ the vibration resonant depolarization ratio.

The vibrational depolarization ratio $\rho^{\mathbf{R}}$ can be expressed in terms of the CARS analogues $\bar{\alpha}^{2}, \bar{\gamma}_{s}{ }^{2}$, and $\bar{\gamma}_{a}{ }^{2}$ of the isotropic and anisotropic Raman tensor invariants $\left(\alpha^{2}, \gamma_{s}^{2}\right.$, and $\left.\gamma_{a}^{2}\right):^{2,14}$

$$
\rho^{\mathrm{R}}=\frac{\chi_{1221}^{(3) \mathrm{R}}}{\chi_{1111}^{(3) \mathrm{R}}}=\frac{-5 \bar{\gamma}_{a}^{2}+3 \bar{\gamma}_{s}{ }^{2}}{45 \bar{\alpha}^{2}+4 \bar{\gamma}_{s}{ }^{2}} .
$$

From Eq. 7 it follows that with the negligible antisymmetric part $\left(\bar{\gamma}_{a}^{2}=0\right)$, the CARS depolarization ratio $\rho^{\mathbf{R}}$ corresponds to the spontaneous Raman depolarization ratio, defined by $\rho_{\mathrm{RS}}=I_{\perp} / I_{\|}$.

A general expression for the measured PS CARS intensity is

$$
\begin{gathered}
I_{A}\left(\omega_{a}\right) \sim \mid \vec{e}_{A}^{*} \cdot\left(\chi_{111}^{(3) \mathrm{NR}}+\chi_{1111}^{(3) \mathrm{E}}+\chi_{1111}^{(3) \mathrm{R}}\right) \vec{e}_{1}\left(\vec{e}_{1} \cdot \vec{e}_{2}^{*}\right) \\
+\left(\chi_{1221}^{(3) \mathrm{NR}}+\chi_{1221}^{(3) \mathrm{E}}+\chi_{1221}^{(3) \mathrm{R}}\right) \\
\quad \times\left.\left(\vec{e}_{2}^{*}-\vec{e}_{1}\left(\vec{e}_{1} \cdot \vec{e}_{2}^{*}\right)\right)\right|^{2} I_{1}^{2} I_{2}
\end{gathered}
$$

where $I_{1}$ and $I_{2}$ denote intensities of the fields at frequencies $\omega_{1}$ and $\omega_{2}$.

\section{EXPERIMENTAL}

The optical layout of the CARS spectrometer is shown in Fig. 2. The second harmonic of the $\mathrm{Nd}$ : YAG laser (operated at $10 \mathrm{~Hz}$ ) is used to provide a pump beam at $\lambda_{\text {pu }}=532 \mathrm{~nm}$, with pulse energy $E_{\mathrm{pu}}=1 \mathrm{~mJ}$, and two CARS probe beams at $\lambda_{1}=532 \mathrm{~nm}, E_{1}=40 \mu \mathrm{J}$, and $\lambda_{2}$ $=578-585 \mathrm{~nm}$ (rhodamine 110 dye laser), $E_{2}=140 \mu \mathrm{J}$. The resulting CARS signal is at $\lambda_{a}=493-488 \mathrm{~nm}$. The positions of these wavelengths are depicted in the $a b$ sorption spectrum of rhodamine $6 \mathrm{G}$ in Fig. 3A. The struc-

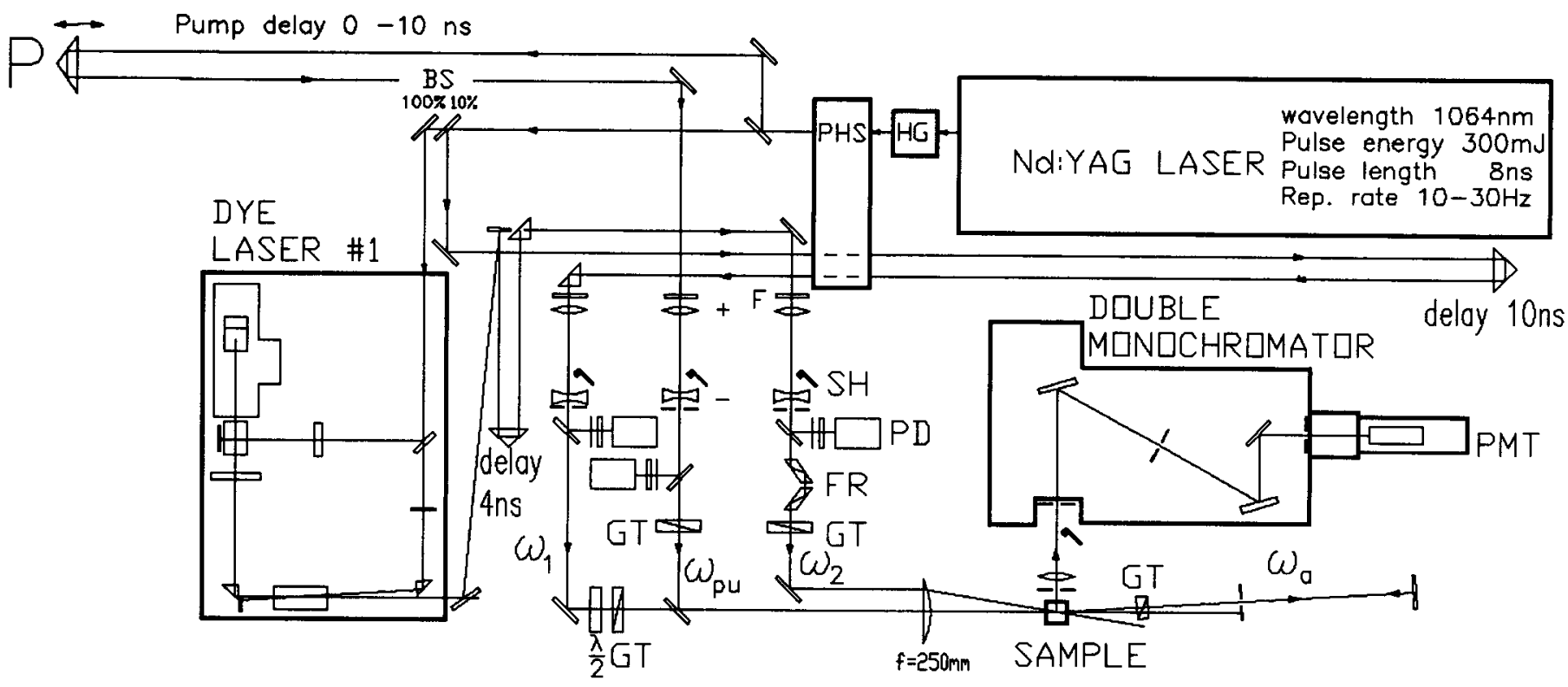

FIG. 2. Experimental setup of the CARS spectrometer. HG, harmonic generator; PHS, prism harmonic separator; BS, beamsplitter; $\mathbf{P}$, prism used for time delay; F, neutral-density filter; SH, shutter; PD, PIN photodiode; FR, double Fresnel-Rhomb; GT, Glan-Taylor polarizer; PMT, photomultiplier tube. 

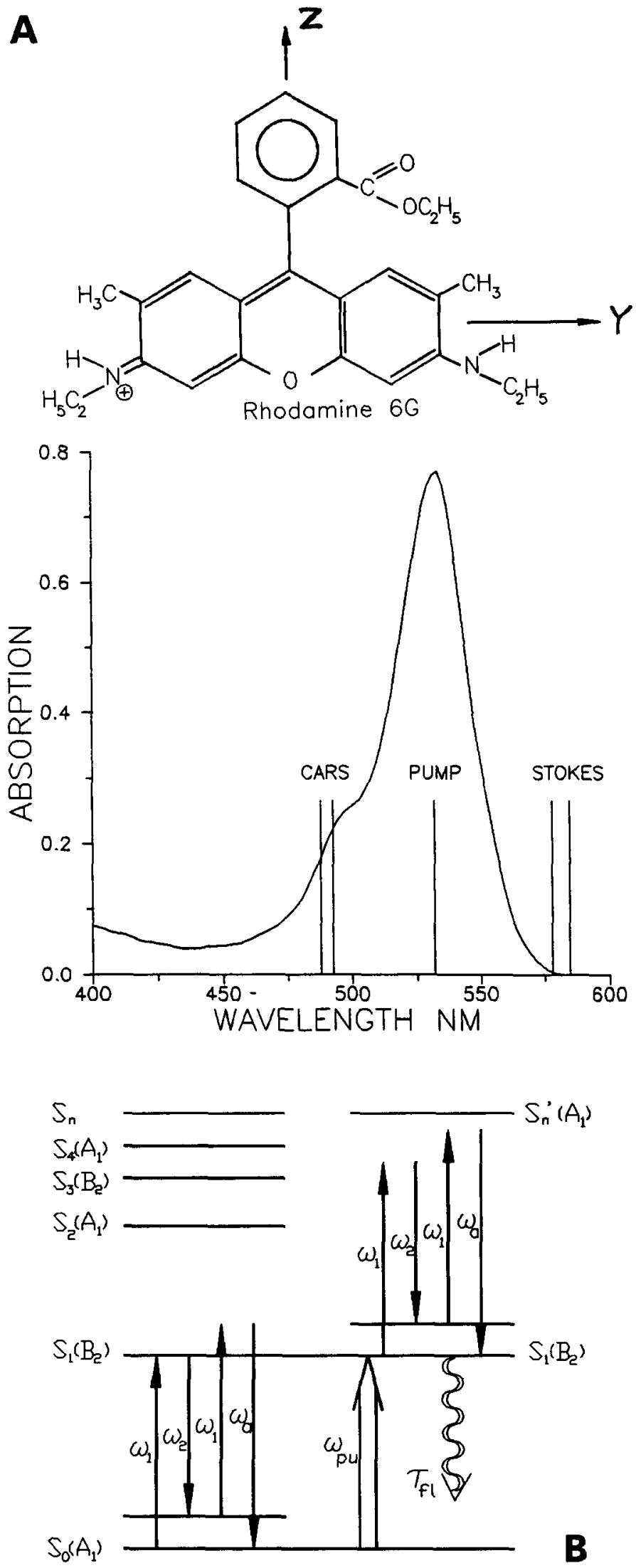

FIG. 3. (A) Molecular structure and absorption spectrum of $2.5 \cdot 10^{-4}$ $\mathbf{M}$ rhodamine 6G in ethanol. The cuvet had a length of $0.25 \mathrm{~mm}$ and antireflection coated windows. Excitation wavelength and CARS probe and signal wavelengths are indicated by vertical lines. (B) Energy schemes corresponding to the CARS process.

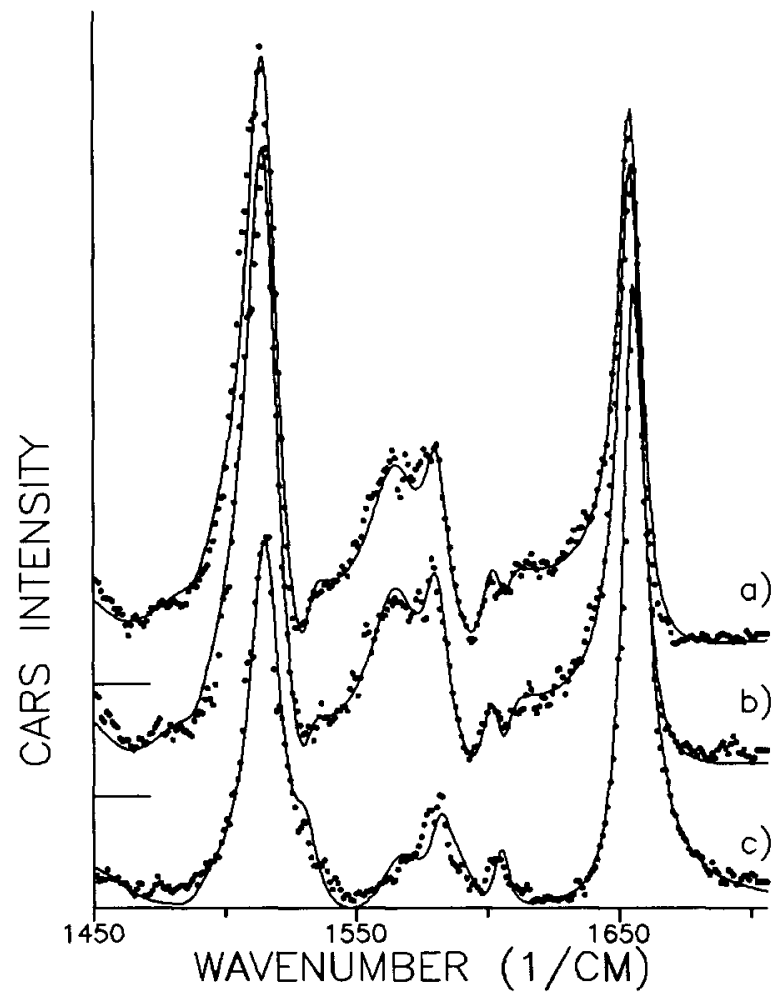

FIg. 4. Polarization resonance CARS spectra of ground-state $2.5 \cdot 10^{-4}$ M rhodamine 6G in ethanol with polarization conditions $\phi\left(\vec{e}_{1}, \vec{e}_{2}\right)=$ $45^{\circ}$ :
(a) $\vec{e}_{A} \| \vec{e}_{1}$
(b) $\vec{e}_{A} \perp \vec{e}_{1}$
$I_{a} \sim\left|\chi_{1111}^{(3) N R}+\chi_{1111}^{(3) R}\right|^{2}$
(c) $\vec{e}_{A} \perp \vec{P}^{\mathrm{NR}}$
$I_{a} \sim\left|\chi_{2112}^{(3) N R}+\chi_{2112}^{(3) R}\right|^{2}$
$I_{a} \sim \mid \sin \phi^{\mathrm{NR}} \chi_{1111}^{(3) \mathrm{R}}$
$-\left.\left(\sin \phi^{\mathrm{NR}}+\sin \left(\phi-\phi^{\mathrm{NR}}\right)\right) \chi_{2112}^{(3, \mathrm{R}}\right|^{2}$.

Experimental data are given by dots; the solid line is the fit result with parameters given in Table II. The scale of $b$ and $c$ are $4 \times$ and $15 \times$ that of a, respectively. The horizontal lines indicate zero intensity.

ture and some transition dipole moment orientations in the rhodamine $6 \mathrm{G}$ molecule are also given in this figure. Energy schemes involved in the CARS process are given in Fig. 3B. With spectral bandwidths of the lasers $\Delta \omega_{1}$ $<1 \mathrm{~cm}^{-1}$ and $\Delta \omega_{2} \simeq 0.25 \mathrm{~cm}^{-1}$, the resulting spectral resolution (assuming Gaussian envelopes) is $\Delta \omega_{a} \leq 1.4$ $\mathrm{cm}^{-1}$. Pulse durations are $8 \mathrm{~ns}$. The delay between pump and probe pulses is adjusted with prism $P$ and can be varied from 0 to $10 \mathrm{~ns}$. Polarization directions of the beams are adjusted by Glan-Taylor polarizing prisms and are indicated in Fig. 1. The focus diameters of the probe beams are $150-250 \mu \mathrm{m}$, and of the pump beam $\geq 250$ $\mu \mathrm{m}$. This configuration ensures that the CARS probing volume is completely inside a region with excited molecules. Overlap of the beams is checked visually with a microscope. The generated CARS signal is analyzed by a Glan-Taylor polarizer and collected by a spherical mirror on a rotation mount with the axis of rotation coinciding with the vertical axis of the cuvet. The signal is deflected onto a mirror on this axis and focused on the entrance slit of a double monochromator (UV-Vis 200 $\mathrm{mm}$, Jobin-Yvon) and is detected by a photomultiplier tube (RCA 9973B). The computer and electronics are interfaced by an IEEE bus. The CARS signals are digitized in an 8-channel 12-bit analog-to-digital converter 
(ADC) and stored on floppy disk for later analysis. Due to competition of CARS signal generation and absorption of the incident and CARS beams in the case of absorbing media, an optimum sample length exists for maximum CARS signal. The optimum sample length $L_{\text {opt }}$ at a concentration $N$ is given by ${ }^{15} \ln \left(\left(2 \alpha_{1}+\alpha_{2}\right) / \alpha_{a}\right) /(\Delta \alpha)$ where $\alpha_{1}, \alpha_{2}$, and $\alpha_{a}$ are the exponential absorption coefficients of the pump, Stokes, and CARS beams at concentration $N$, and $\Delta \alpha=\left(2 \alpha_{1}+\alpha_{2}-\alpha_{a}\right) / 2$. These absorption coefficients are related to the measured absorption by $\alpha_{\lambda}(N)$ $=2.3 A_{\lambda}(N) / L$, with $A_{\lambda}(N)$ the measured absorbance at wavelength $\lambda$ and concentration $N$, and $L$ the length. From Fig. 3A it follows that $L_{\text {opt }}\left(\right.$ at $\left.1450 \mathrm{~cm}^{-1}\right)=0.32$ $\mathrm{mm}$ and $L_{\mathrm{opt}}\left(\right.$ at $\left.1700 \mathrm{~cm}^{-1}\right)=0.34 \mathrm{~mm}$ at concentration rhodamine 6G $N=2.5 \cdot 10^{-4} \mathrm{M} / \mathrm{L}$. The sample flow cuvet in our setup has a length of $0.25 \mathrm{~mm}$, which is close to this optimum. Cuvet windows are $0.15 \mu \mathrm{m}$ thick and have been anti-reflection coated on the air/glass interfaces to minimize multiple interference effects. ${ }^{16} \mathrm{~A}$ peristaltic pump refreshes the sample after each pulse. Spectra are measured in scanning mode with $\omega_{1}$ fixed and with automated phase-match adjustment by varying the distance between the two parallel beams on a $250-\mathrm{mm}$ lens. A spectral range 1500 to $1700 \mathrm{~cm}^{-1}$ is recorded in 250 points, with 30 pulses averaged per point. All spectra shown were corrected for laser intensity variations in this range and smoothed by a 5-point Savitsky-Golay smoothing procedure. ${ }^{17}$

\section{RESULTS}

Ground State $\left(S_{0}\right)$ and Excited Singlet State $\left(S_{1}\right)$. Figure 4 shows the ground-state polarization CARS spectra (dots) with polarization conditions (a) $\vec{e}_{A} \| \vec{e}_{1}$ where a $\chi_{1111}^{(3)}$ contribution is measured; (b) $\vec{e}_{A} \perp \vec{e}_{1}$ with a $\chi_{1221}^{(3)}$ $\left(=\chi_{212}^{(3)}\right.$ in isotropic ground state) contribution; and (c) $\vec{e}_{A} \perp \vec{P}^{\mathrm{NR}}$ at suppression of the nonresonant background. The Raman bands observed here are from $S_{0}$ rhodamine 6G. Ethanol has vibrations at 1455 and $1479 \mathrm{~cm}^{-1}$, but these are weak in comparison with the resonantly enhanced rhodamine vibrations. The background arises mainly from the nonvibration resonant contribution $\chi_{\mathrm{E} t h}^{(3) \mathrm{NR}}$ of ethanol.

In Fig. 5 the same polarization conditions were applied as in Fig. 4, but here the leading edge of the $\omega_{\mathrm{pu}}$ excitation pump pulses preceded that of the CARS probe pulses by $1.5 \mathrm{~ns}$. Since we define the " 1 " polarization direction along the $\vec{e}_{\mathrm{pu}}\left(=\vec{e}_{1}\right.$ in our case), we measure "1111" and "2112" components in the case pump pulses are applied.

Besides the depletion of the ground-state vibration at $1517 \mathrm{~cm}^{-1}$, strong bands at 1532 and $1606 \mathrm{~cm}^{-1}$ appear, attributed to the excited singlet state $S_{1}{ }^{7}$ The high pedestal is caused by the pure electronic resonant contribution $\chi_{S_{1}}^{(3)}$ of the excited $S_{1}$ state. Below, it is shown that the electronic contribution from ground-state rhodamine $6 \mathrm{G} \chi_{S_{0}}^{(3) \mathrm{E}}$ is negligible. In Figs. 4 and 5, the dots represent measured data and the solid line is the result of a simultaneous fit of spectra $4 a, 4 b$, and $4 c$, and $5 a$ and $\mathbf{5 b}$, respectively (see section below).

Variation of the Pump-Beam Polarization. The effect of applying different linear polarizations of the pre-excitation pump laser beam $\left(\vec{e}_{\mathrm{pu}}\right)$ with respect to the polarization orientation of the CARS probe laser beams $\left(\vec{e}_{1}\right.$

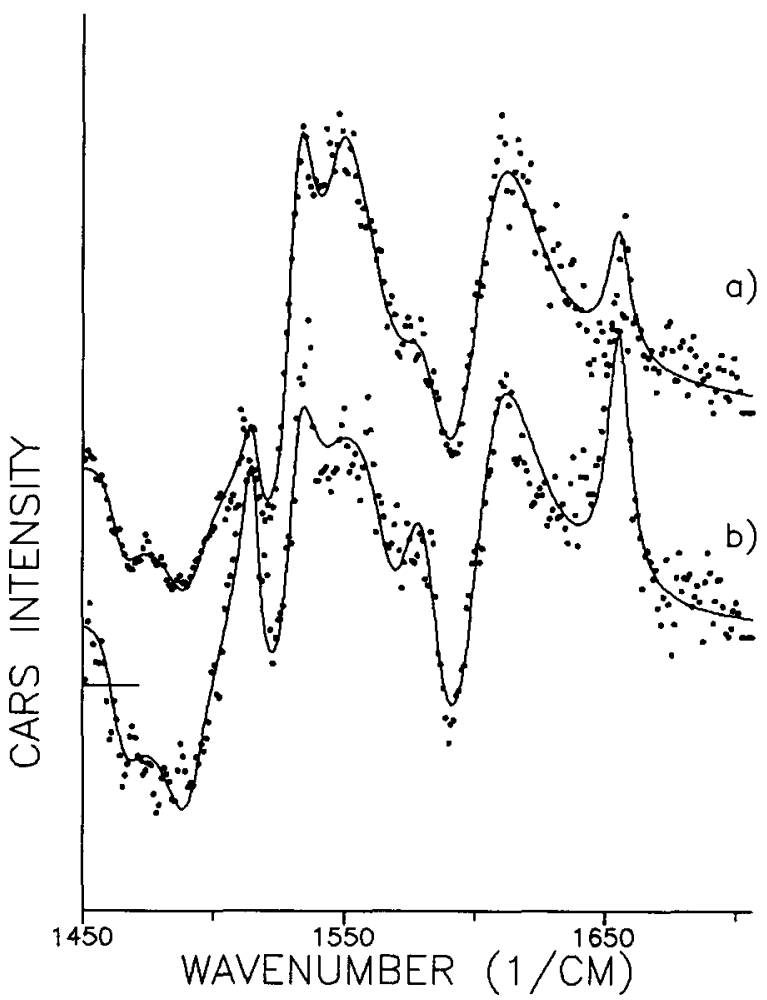

FIG. 5. Polarization CARS spectra of excited state $2.5 \cdot 10^{-4} \mathrm{M}$ rhodamine $6 \mathrm{G}$ in ethanol with pre-excitation pulse $\left(\lambda_{\mathrm{pu}}=532 \mathrm{~nm}\right) 1.5 \mathrm{~ns}$ before the CARS probe pulses. Polarization conditions with $\phi\left(\vec{e}_{1}, \vec{e}_{2}\right)=45^{\circ}, \vec{e}_{\text {pu }} \| \vec{e}_{1}$ :
(a) $\vec{e}_{A} \| \vec{e}_{1} \quad I_{a} \sim\left|\chi_{1111}^{(3) N R}+\chi_{1111}^{(3) E}+\chi_{1111}^{(3) R}\right|^{2}$
(b) $\vec{e}_{A} \perp \vec{e}_{1} \quad I_{a} \sim\left|\chi_{212}^{(3) N R}+\chi_{2112}^{(3) E}+\chi_{2112}^{(3) R}\right|^{2}$.

Experimental data are given by dots; solid lines are fit result with parameters given in Table III. The scale of $\mathbf{b}$ is $1 / 7 \times$ that of $\mathbf{a}$.

and $\vec{e}_{2}$ ) is demonstrated in Fig. 6. Here again, the leading edge of the $\omega_{\mathrm{pu}}$ excitation pump pulses preceded that of the CARS probe pulses by $1.5 \mathrm{~ns}$. With parallel pump and CARS probe polarization $\left(\vec{e}_{\mathrm{pu}}\left\|\vec{e}_{1}\right\| \vec{e}_{\mathrm{A}}\right)$, a $\chi_{111}^{(3)}$ contribution is measured that is larger than in the case of perpendicular polarizations $\left(\vec{e}_{\mathrm{pu}} \perp \vec{e}_{1} \| \vec{e}_{A}\right)$ where a $\chi_{2222}^{(3)}$ contribution is measured. For the isotropic case these two spectra should be identical. The difference in the spectra indicates that the orientation distribution of transition dipole moments in molecules excited to the $S_{1}$ state is anisotropic on a nanosecond time scale. This observation may be due to photoselection of molecules with an absorption moment (almost) parallel to the polarization of the pump and/or orientation of the molecules in the direction of the (strong) pump field polarization by the interaction of induced dipole moments with the electric field. The orientation distribution function is $f(\theta)=\cos ^{2} \theta$ where $\theta$ denotes the angle between the pump-polarization axis and the transition dipole moment of the molecule. For the isotropic case this function would be $f(\theta) \equiv 1$.

From the differences in the spectra measured with different pump polarization orientations it follows that the effect of rotational diffusion-relaxation (or reorientation) of the rhodamine $6 \mathrm{G}$ molecules in ethanol is small during the overlap time of pump and CARS probe pulses. Since the reorientational diffusion time of rhodamine $6 \mathrm{G}$ 


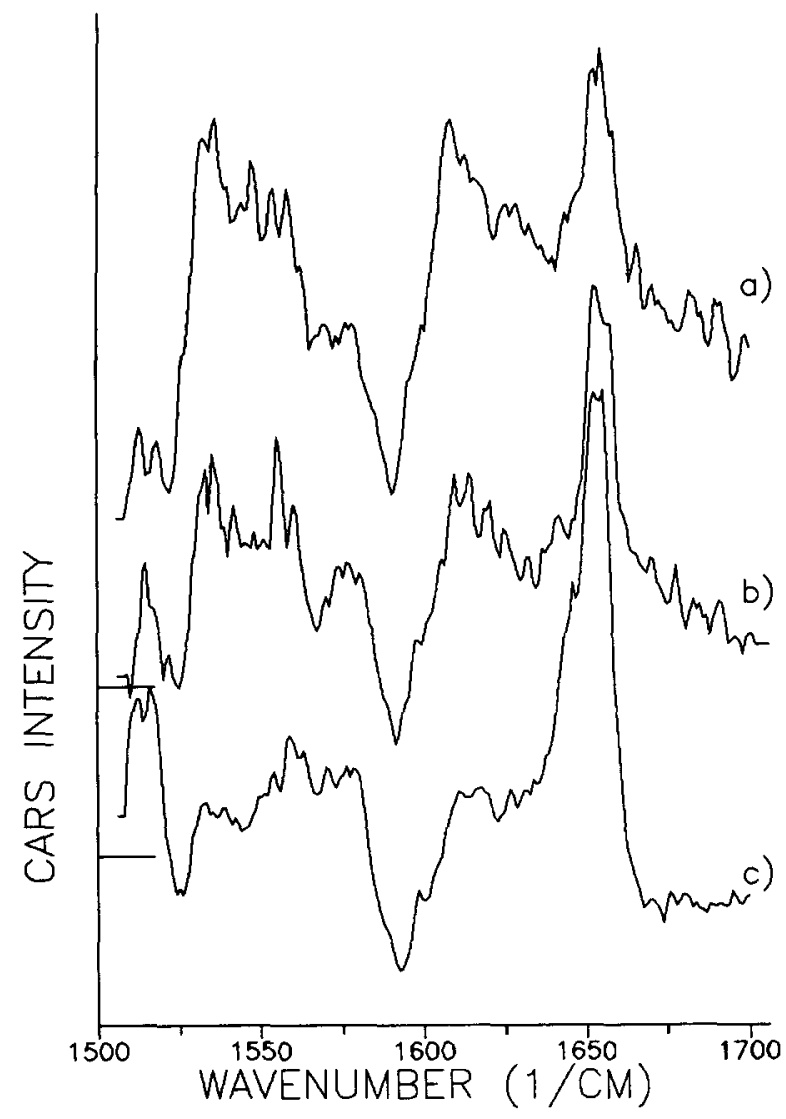

FIG. 6. CARS spectra of the excited state of $2.510^{-4} \mathrm{M}$ rhodamine $6 \mathrm{G}$ in ethanol with pre-excitation pulse $\left(\lambda_{p u}=532 \mathrm{~nm}\right) 1.5 \mathrm{~ns}$ before CARS probe pulses with different polarizations $\vec{e}_{\mathrm{pu}}$ with respect to the polarizations $\vec{e}_{1}$ and $\vec{e}_{2}$ of the CARS probe beams, with $\phi\left(\vec{e}_{1}, \vec{e}_{2}\right)=$ $45^{\circ}, \vec{e}_{A} \| \vec{e}_{1}$, and $\chi_{i j k l}^{(3)}=\chi_{i j k l}^{(3) \mathrm{NR}}+\chi_{i j k l}^{(3) \mathrm{E}}+\chi_{i j k l}^{(3) \mathrm{R}}$ :
(a) $\vec{e}_{\mathrm{pu}} \| \vec{e}_{1} \quad I_{a} \sim\left|\chi_{1111}^{(3)}\right|^{2}$
(b) $\vec{e}_{\mathrm{pu}} \| \vec{e}_{2} \quad \dot{I}_{a} \sim\left|\chi_{1111}^{(3)}+\chi_{1221}^{(3)}+\chi_{2121}^{(3)}+\chi_{2211}^{(3)}\right|^{2}$;
(c) $\vec{e}_{\mathrm{pu}} \perp \vec{e}_{2} \quad I_{a} \sim\left|\chi_{2222}^{(3)}\right|^{2}$.

The scale of $\mathbf{b}$ is twice that of $\mathbf{a}$ and $\mathbf{c}$.

in ethanol was estimated (in a picosecond pulse experiment) to be $\tau_{\text {rot }}=0.27 \mathrm{~ns},{ }^{12}$ this means that the excited rhodamine $6 \mathrm{G}$ molecules maintain their orientation during the pump-probe pulses overlap time. This behavior may be explained by the discussion that follows.

In the absence of the strong pump laser beam the spontaneous $S_{1}$ lifetime is $4.5 \mathrm{~ns}$. In the presence of the pump beam the $S_{0}$ lifetime is shortened by depletion of the ground state through absorption. The excited $S_{1}$ state lifetime is shortened (from $4.5 \mathrm{~ns}$ to about $2 \mathrm{~ns}$ ) by stimulated emission from the $S_{1}$ to the $S_{0}$ state $^{12}$ and also by depletion of the $S_{1}$ state through absorption from the $S_{1}$ state to higher-energy states $S_{n}$. The transition rates of these processes depend on the pump laser beam intensity. The effective shortening of the $S_{1}$ lifetime somewhat lowers the effect of reorientational diffusion. However, since many photons are generated during the pump pulse which excite rhodamine $6 \mathrm{G}$ molecules to the $S_{1}$ state and create anisotropy along the pump beam polarization in the molecular distribution of both the $S_{0}$ and $S_{1}$ state, the induced anisotropy is expected to last as long as the pump pulse duration. When the pump pulse is over, the anisotropic distribution quickly $\left(\tau_{\text {rot }}\right)$ reorients to an iso- tropic distribution. The contribution from the 1.5-ns part of the CARS probe pulse tails that don't overlap with the pump pulse (in which the molecules may reorient) probably is negligible compared with that of the former part because of the lower energies in the tails of the CARS probe pulses. The distribution of the optically induced anisotropy of the excited state is assumed to be ${ }^{18}$

$$
f_{\mathrm{S}_{1}}(\theta)=N_{1} \cos ^{2} \theta
$$

which also causes the ground-state distribution to be changed from isotropic (spherically symmetric) to anisotropic

$$
f_{S_{0}}(\theta)=N_{0}-N_{1} \cos ^{2} \theta=N_{0}\left(1-g_{1} \cos ^{2} \theta\right)
$$

where $N_{0}$ and $N_{1}$ denote the ground-state and excitedstate concentration, and $g_{1}=N_{1} / N_{0}$ represents the excited fraction. The anisotropic distribution of excited molecules imposes different relations between the components of the third-order nonlinear susceptibility tensor $\chi_{i j k l}^{(3) E}$ of the solution with $S_{1}$ excited molecules and molecules in the ground state $S_{0}$. The anisotropy of both ground state and excited state can thus lead to changes of the observed vibrational depolarization ratios in the presence of an excitation pulse. The relations between the (macroscopic) $\chi_{i j k l}^{(3) E}$ components in the ground state $S_{0}$ or excited state $S_{1}$ can now be derived from orientational averaging of the molecular susceptibility $\chi^{(3, \text { mol)E }}$ with the appropriate anisotropic distribution functions $f_{S_{1,0}}(\theta)$ defined in Eqs. 9 and 10. These relations are derived in Appendix A. Assuming parallel transition dipole moments for the CARS process in the molecule and $g_{1}$ $=1$, this approach results in the following relations between $\chi^{(3) \mathrm{E}}$ components (see Appendix A):

$$
\chi_{1111}^{(3) \mathrm{E}}: \chi_{2222}^{(3) \mathrm{E}}: \chi_{1221}^{(3) \mathrm{E}}: \chi_{1122}^{(3) \mathrm{E}}: \chi_{2112}^{(3) \mathrm{E}}: \chi_{2211}^{(3) \mathrm{E}}=5: 1: 1: 1: 1: 1 \text {. }
$$

Concentration-Dependent Measurements. Under electronic resonance conditions the electronic susceptibility $\chi_{S_{0}}^{(3)}$ of the rhodamine $6 \mathrm{G}$ molecules may become comparable to that of the (nonresonant) electronic susceptibility $\chi_{\mathrm{Eth}}^{(3) \mathrm{NR}}$ of the solvent molecules, particularly when the concentration of rhodamine $6 \mathrm{G}$ is high. ${ }^{19}$

In order to distinguish between the electronic susceptibilities of ground-state rhodamine $6 \mathrm{G}$ and solvent, a series of rhodamine $6 \mathrm{G}$ concentrations in ethanol was examined. The measurements were carried out with the use of the flow cuvet and a reservoir with the rhodamine $6 \mathrm{G}$ in ethanol solution. The concentration was changed by adding ethanol to the reservoir. The sample length was kept constant at $0.25 \mathrm{~mm}$. The spectra recorded under these conditions are shown in Fig. 7, with concentration $N_{j}$ ranging from $5 \cdot 10^{-4}$ to $1.67 \cdot 10^{-5} \mathrm{M} / \mathrm{L}$. The spectra were fitted according to $I_{a}(x) \sim\left|\chi^{(3)}(x)\right|^{2}$ with $\chi^{(3)}(x)$ given in Eq. 6 under the assumption of $g_{s}=0$ with $s \neq 0$ (i.e., only the ground state is populated) and $N_{\text {Sol }}=1$ :

$$
\begin{aligned}
\chi_{j}^{(3)}(x)=\left[\chi_{\mathrm{Eth}}^{(3) \mathrm{NR}}+\sum_{t} \frac{\gamma_{t \mathrm{Eth}}^{(3) \mathrm{R}}}{\left(\Omega_{t \mathrm{Eth}}^{\mathrm{R}}-x\right) / \Gamma_{t \mathrm{Eth}}^{\mathrm{R}}-i}\right. \\
+N_{0 j}\left(\frac{\gamma_{S_{0}}^{(3) \mathrm{E}} \exp \left(i \theta_{0}^{\mathrm{E}}\right)}{\left(\Omega_{0}^{\mathrm{E}}-x\right) / \Gamma_{0}^{\mathrm{E}}-i}\right.
\end{aligned}
$$




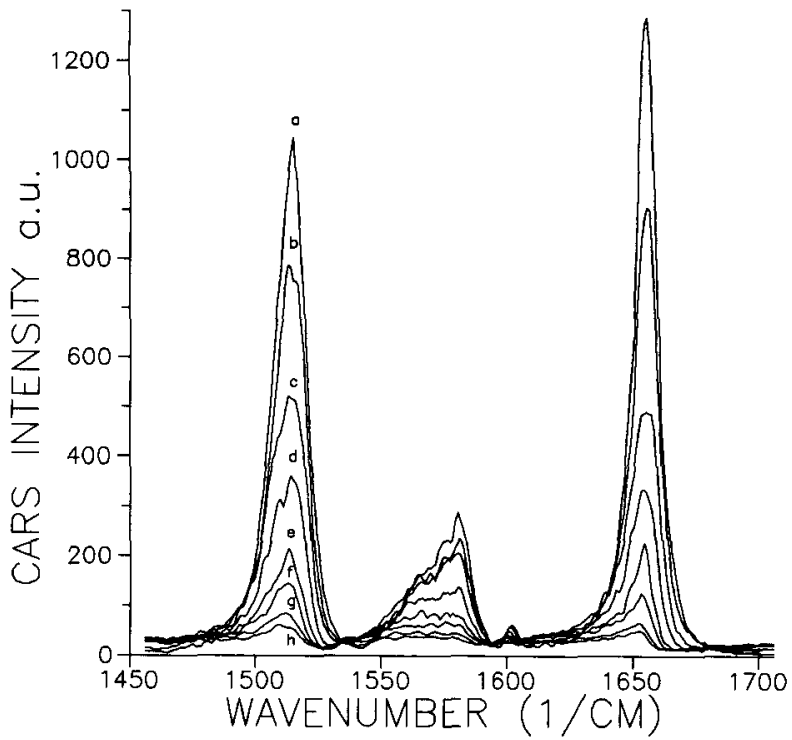

FIG. 7. CARS spectra of different concentrations (in $\mathbf{M} / \mathrm{L}$ ) rhodamine $6 \mathrm{G}$ in ethanol: (a) $5.0 \cdot 10^{-4}$; (b) $2.5 \cdot 10^{-4}$; (c) $1.25 \cdot 10^{-4}$; (d) $8.33 \cdot 10^{-5}$; (e) $5.0 \cdot 10^{-5}$; (f) $3.57 \cdot 10^{-5} ;$ (g) $2.27 \cdot 10^{-5}$; (h) $1.67 \cdot 10^{-5}$. The spectra are rescaled to correct for absorption. Fit results are listed in Table I.

$$
\left.\left.+\sum_{t} \frac{\gamma_{0 t}^{(3) \mathrm{R}} \exp \left(i \theta_{0 t}^{\mathrm{R}}\right)}{\left(\Omega_{0 t}^{\mathrm{R}}-x\right) / \Gamma_{0 t}^{\mathrm{R}}-i}\right)\right] c_{j} .
$$

Since the frequency dependence of the electronic contribution is small in the range 1450 to $1700 \mathrm{~cm}^{-1}$, a large electronic bandwidth $\Gamma_{0}^{\mathrm{E}}=500 \mathrm{~cm}^{-1}$ was used with $\Omega_{0}^{\mathrm{E}}$ at the center of the spectral range. In Eq. $12 j$ indentifies different concentrations and $c_{j}$ is a scaling parameter that accounts for differences in absorption for each concentration $N_{0}$. The set of eight spectra was simultaneously fitted with a single set of Raman band parameters $\left(\Omega_{s t}^{\mathrm{R}}, \Gamma_{s t}, \gamma_{s t}^{\mathrm{R}}\right.$ and $\theta_{s t}^{\mathrm{R}}$ for each vibration $\left.t\right)$; concentrations $N_{0 j}$ and scaling parameters $c_{j}$ were free parameters. Two different fits were performed-the first with $\gamma_{S_{0}}^{(3) E}=0$, and thus only a real solvent susceptibility $\chi_{\mathrm{Eth}}^{(3) \mathrm{NR}}$, and the second with $\chi_{\mathrm{Eth}}^{(3) \mathrm{NR}}, \gamma_{\mathrm{S}_{0} \mathrm{E}}^{(3)}$, and $\theta_{0}^{\mathrm{E}}$ as free parameters. The fit results are listed in Table I.

From Eq. 12 it can be seen that if $\left|\gamma_{S_{0}}^{(3) \mathrm{E}}\right| \gg$ $\chi_{\mathrm{Eth}}^{(3) \mathrm{NR}} / N_{0 j}$, no spectral line shape changes should be observed for different concentrations, since the intensity would then be proportional to $N_{0 j}^{2}\left|\gamma_{S_{0}}^{(3) \mathrm{E}}+\gamma_{S_{0}}^{(3) \mathrm{R}}\right|^{2}$. On the other hand if $\left|\gamma_{S_{0}}^{(3) E}\right| \ll \chi_{\mathrm{Eth}}^{(3) \mathrm{NR}} / N_{\mathrm{o}}$, the line shapes should change due to interference of $\chi_{\mathrm{Eth}}^{(3) \mathrm{NR}}$ with $N_{0 j} \gamma_{S_{0}}^{(3) \mathrm{R}}$ when varying $N_{0 j}$. The latter applies to our case since in Fig. 7 line shape changes are observed, viz., dispersive line shapes at low concentrations and almost Lorentzian line shapes at higher concentrations. The fit results given in Table I for the two models are almost identical, and the fitted values for the concentrations are close to the ones calculated from the dilution. From this observation we conclude that the influence of $\gamma_{S_{0}}^{(3) E}$ is small relative to $\chi_{\mathrm{Eth}}^{(3) \mathrm{NR}}\left(\right.$ ratio $N_{0} \gamma_{S_{0}}^{(3) \mathrm{E}} / \chi_{\mathrm{Eth}}^{(3) \mathrm{NR}}=0.16$ for $\left.N_{0}=2.5 \cdot 10^{-4} \mathrm{M} / \mathrm{L}\right)$. The ground-state electronic susceptibility $\gamma_{S_{0}}^{(3) E}$ of rhodamine $6 \mathrm{G}$ will thus be neglected in further analyses.

Coherent Ellipsometry of the Electronic Susceptibility. The coherent ellipsometry technique ${ }^{20}$ allows measure-
TABLE I. Concentration fit parameters $N_{\text {fit }}$ (without $\chi_{S_{0}}^{(3) E}$ ) and $N_{\text {fit }}$ (with $\chi_{S_{0}}^{(3)}$ ) of rhodamine 6G in ethanol (Fig. 7).

\begin{tabular}{cccc}
\hline Spectrum no. & $\begin{array}{c}N_{\text {calc }} \\
\left(10^{-5} \mathbf{M} / L\right)\end{array}$ & $\begin{array}{c}N_{\text {fit1 }} \\
\left(10^{-5} \mathbf{M} / L\right)\end{array}$ & $\begin{array}{c}N_{\text {fit2 }} \\
\left(10^{-5} \mathbf{M} / L\right)\end{array}$ \\
\hline 1 & 50.0 & 50.0 & 50.0 \\
2 & 25.0 & 25.0 & 25.0 \\
3 & 12.5 & 12.5 & 12.5 \\
4 & 8.33 & 8.10 & 8.10 \\
5 & 5.00 & 5.03 & 5.03 \\
6 & 3.57 & 3.61 & 3.60 \\
7 & 2.27 & 2.12 & 2.07 \\
8 & 1.67 & 1.51 & 1.46
\end{tabular}

${ }^{a}$ Calculated concentration from diluting the sample solution. Fit 2: $\chi_{s_{0}}^{\mathrm{E}} / \chi_{\mathrm{Eth}}^{(3) \mathrm{NR}}=0.16$.

ment of both real and imaginary parts of the resonant susceptibility $\chi_{i j k l}^{(3) E}$. The polarization $P^{\mathrm{E}}$ is given by

$$
\vec{e}_{A}^{*} \cdot \vec{P}^{\mathrm{E}}\left(\omega_{a}\right) \sim \vec{e}_{A}^{*} \cdot \chi_{1111}^{(3) E}\left(\cos \phi \vec{e}_{x}+\rho^{\mathrm{E}} \sin \phi \vec{e}_{y}\right) E_{1}^{2} E_{2}^{*}
$$

where $\vec{e}_{1}=\vec{e}_{x}$, and $\vec{e}_{2}=\cos \phi \vec{e}_{x}+\sin \phi \vec{e}_{y}$, and $\phi$ is the polarization angle between the probing beams $\omega_{1}$ and $\omega_{2}$. In the case of resonant excitation to the $S_{1}$ state, the $\chi_{S_{1}}^{(3) E}$ contribution can be considered to originate from a linear polarization $\vec{P}^{\mathrm{E}}$ with phase shift $\theta^{\mathrm{E}}$ to the nonresonant contribution $\vec{P}^{\mathrm{NR}}$ from the solvent ethanol, at an angle $\varphi^{\mathrm{E}}=\phi^{\mathrm{E}}-\phi^{\mathrm{NR}}$. These two sources give rise to elliptically polarized CARS signals. Coherent ellipsometry was applied in the following way: At frequency $\omega_{a}=$ $2 \omega_{1}-\omega_{2}$ with $\left(\omega_{1}-\omega_{2}\right) / 2 \pi c=1625 \mathrm{~cm}^{-1}$, where no distinct Raman bands of rhodamine 6G and ethanol are present, the CARS signal intensity was measured at different polarization analyzer angles $\epsilon$ in the signal beam for both the linearly polarized nonresonant CARS signal (without excitation) and the elliptically polarized CARS signal (with excitation). The angle $\phi$ between the polarizations of the CARS probe beams $\omega_{1}$ and $\omega_{2}$ was set at $45^{\circ}$. The polarization conditions are depicted in Fig. 8. The result is given in Fig. 9, where squares and circles represent the experimental data with and without excitation, respectively.

The elliptically polarized CARS signal can formally be broken down into two linearly polarized waves with amplitudes $P_{\xi}$ and $P_{\eta}$ and phase shift $\delta$ between them, where $\vec{P}_{\xi}$ and $\vec{P}_{\eta}$ are polarized parallel and perpendicular to the $\vec{P}^{\mathrm{NR}}$-axis (see Fig. 9).

The mathematical procedure to derive these amplitudes and phases from the measured ellipse is given in Appendix B.

It follows that there are two possible sets, where the polarizations $\vec{P}_{\xi}$ and $\vec{P}_{\eta}$ have either the opposite or the same phase $\delta$. These sets are depicted in Fig. 10. The two possible sets give, furthermore:

$$
\left.\left.\begin{array}{l}
P_{\xi_{+}}^{\mathrm{E}}=-0.796 \\
P_{\eta}^{\mathrm{E}}=0.086 \\
\beta_{+}=50.7^{\circ}
\end{array}\right\} \text { (Set 1) and } \begin{array}{l}
P_{\xi-}^{\mathrm{E}}=0.356 \\
P_{\eta}^{\mathrm{E}}=0.086 \\
\beta_{-}=20.3^{\circ}
\end{array}\right\} \text { (Set 2). }
$$

The phase shift $\theta^{\mathrm{E}}$ between the nonresonant polarization $\vec{P}^{\mathrm{NR}}$ and the linear polarization $\vec{P}^{\mathrm{E}}$ was found to be $\theta^{\mathrm{E}}=$ $\pm 75^{\circ}$. Now we can calculate the amplitude $\left|\vec{P}^{\mathrm{E}}\right|$, the angles $\varphi \mathrm{E}$ and $\phi^{\mathrm{E}}$, the ratio of imaginary and real parts of $\chi^{(3) \mathrm{E}}$, and the depolarization ratio $\rho^{\mathrm{E}}$ and $\chi_{2112}^{(3) \mathrm{E}} / \chi_{1111}^{(3) \mathrm{E}}$ : 


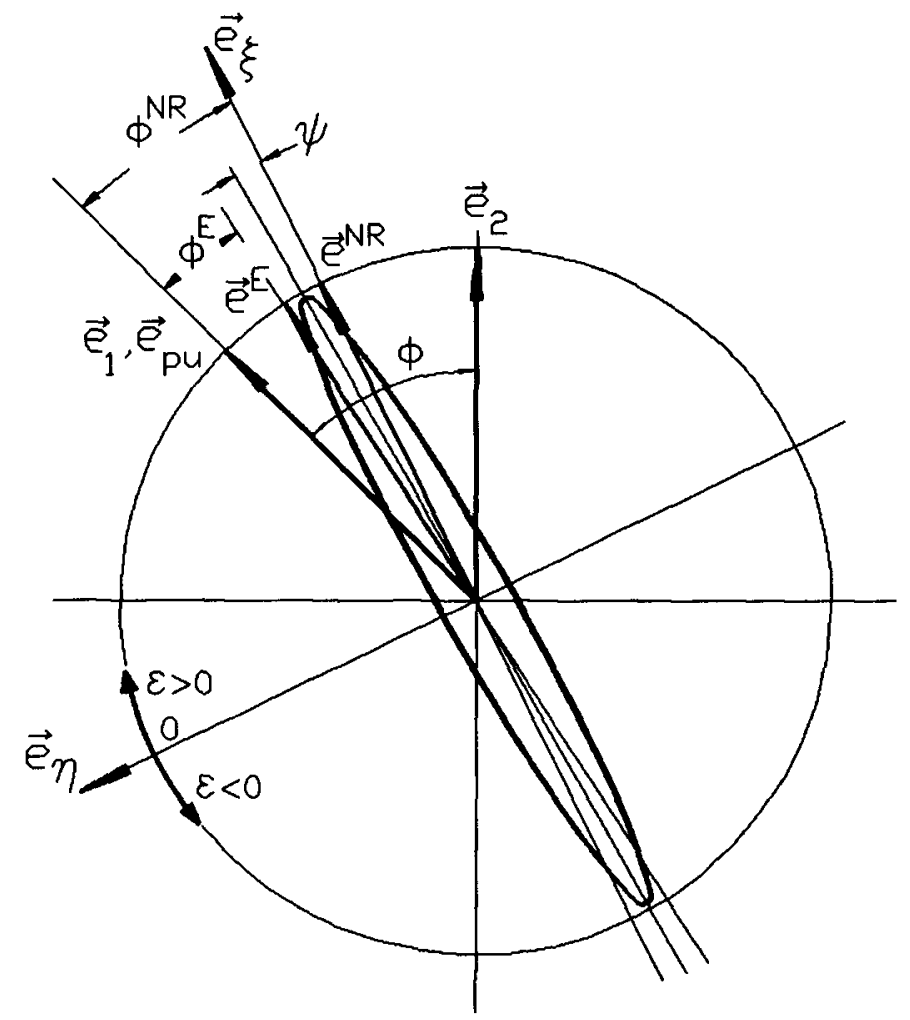

FIG. 8. Polarization orientations of the pump $\vec{e}_{\text {pu }}$, CARS probe $\vec{e}_{1}$ and $\vec{e}_{2}$ as used in the measurements. The $\omega_{2}$ probe polarization is oriented perpendicular to the optical table $\vec{e}_{2}=\vec{e}_{y}$ and makes an angle $\varphi=45^{\circ}$ with the $\omega_{1}$ probe beam polarization. $\vec{e}^{\mathrm{NR}}$ denotes the orientation of nonresonant polarization from the solvent ethanol and $\vec{e}^{E}$ the orientation of resonant polarization due to the $S_{1}$ electronic susceptibility. The ellipse represents the result of the interference between these two linearly polarized waves.

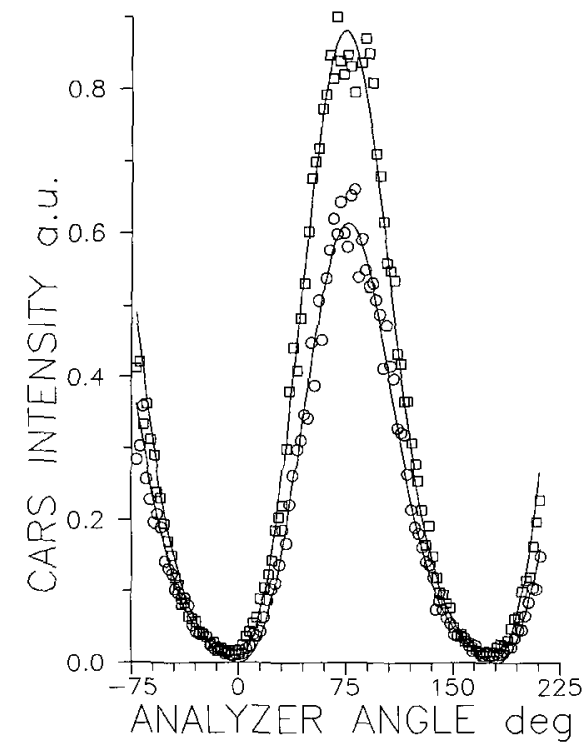

FIG. 9. CARS intensity dependence on the polarization analyzer rotation angle measured at $\left(\omega_{1}-\omega_{2}\right) / 2 \pi c=1625 \mathrm{~cm}^{-1}$ and $\vec{e}_{\mathrm{pu}} \| \vec{e}_{1}$ : (a) unpumped (circles); (b) pumped (squares). The solid curves result from the nonlinear least-squares fits with parameters given in the text.

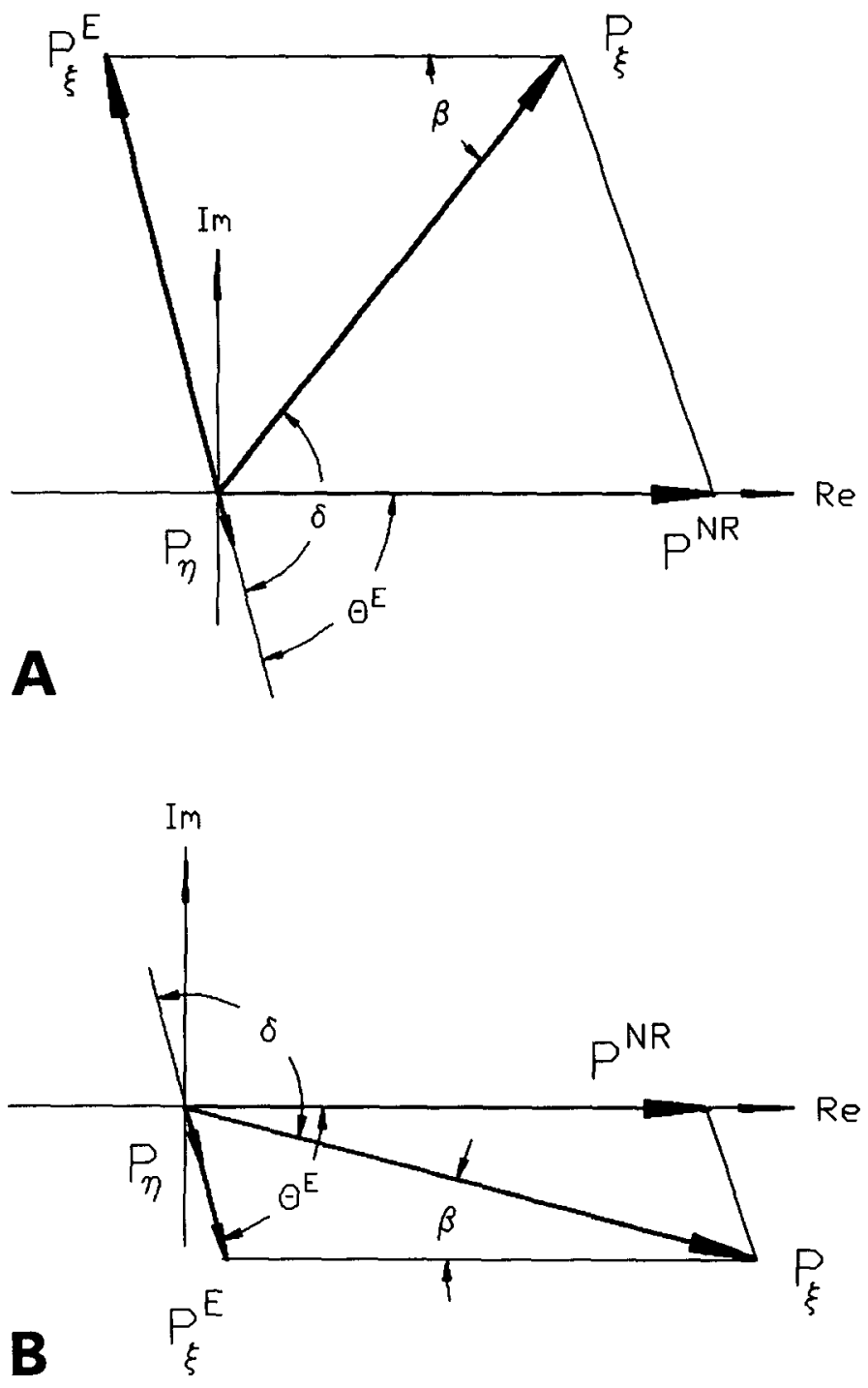

FIg. 10. Representation of the vectors $\vec{P}^{\mathrm{NR}}$ and $\vec{P}^{\mathrm{E}}$ in the complex plane according to the projections of the $\xi$ - and $\eta$-axes. There are two configurations possible where $P_{\xi}^{\mathrm{E}}$ and $P_{\eta}^{\mathrm{E}}$ have opposite phases (A) and the same phase (B).

\section{Set 1}

Set 2

$$
\begin{aligned}
& \left|\vec{P}^{\mathrm{E}}\right|=\sqrt{\left(P_{\xi}^{\mathrm{E}}\right)^{2}+\left(P_{\eta}^{\mathrm{E}}\right)^{2}} \quad(0.80 \pm 0.05) \quad(0.37 \pm 0.05) \\
& \varphi^{\mathrm{E}}=\arctan \left(P_{\eta}^{\mathrm{E}} / P_{\xi}^{\mathrm{E}}\right) \quad(-6 \pm 3)^{\circ} \quad(14 \pm 6)^{\circ} \\
& \phi^{\mathrm{E}}=\varphi^{\mathrm{E}}+\phi^{\mathrm{NR}} \quad(12 \pm 3)^{\circ} \quad(32 \pm 6)^{\circ} \\
& \operatorname{Im} \chi_{i j k l}^{(3) \mathrm{E}} / \operatorname{Re} \chi_{i j k l}^{(3) \mathrm{E}}=\tan \theta^{\mathrm{E}} \pm(3.7 \pm 0.4) \quad \pm(3.7 \pm 0.4) \\
& \rho^{\mathrm{E}}=\tan \phi^{\mathrm{E}} / \tan \phi \quad(0.22 \pm 0.07) \quad(0.6 \pm 0.1) \text {. }
\end{aligned}
$$

In order to distinguish between the two sets we make use of the calculations used above. There it is shown that, under the assumption of parallel transition moments in the molecule $\chi_{1111}^{(3) \mathrm{E}}: \chi_{2112}^{(3) \mathrm{E}}=5: 1$, yielding $\rho^{\mathrm{E}}=0.2$. Furthermore, the high peak originating from the excitedstate contribution $\gamma_{S_{1}}^{(3) \mathrm{E}}$ points to a large value of the ratio $\left|\vec{P}^{\mathrm{E}} / \vec{P}^{\mathrm{NR}}\right|$. In addition, fitting the spectra, we found that the polarized value of $\rho^{\mathbf{E}}$ gives the best result. From these facts we conclude that the first set is correct.

Another method exists to distinguish between the two sets. From the solvent band line shape, one can estimate the phase $\theta^{\mathrm{E}}$ relative to the background phase due to 
TABLE II. Vibrational data of rhodamine 6G without pre-excitation (Fig. 4)."

\begin{tabular}{ccccc}
$\Omega_{0 t}^{\mathrm{R}}\left(\mathrm{cm}^{-1}\right)$ & $\Gamma_{0 t}^{\mathrm{R}}\left(\mathrm{cm}^{-1}\right)$ & $\chi_{0 t}^{(3) \mathrm{R}}($ a.u. $)$ & $\theta_{0 t}^{\mathrm{R}}(\mathrm{deg})$ & $\begin{array}{c}\rho_{0 t}^{\mathrm{R}}= \\
\chi_{2112}^{(3) R} / \chi_{1111}^{(3)}\end{array}$ \\
\hline $1455 \pm 17$ & $20 \pm 9$ & $4 \pm 2$ & $21 \pm 20$ & $0.47 \pm 0.12$ \\
$1489 \pm 9$ & $6 \pm 5$ & $1 \pm 2$ & $74 \pm 50$ & $0.47 \pm 0.30$ \\
$1515.6 \pm 0.9$ & $7.2 \pm 0.8$ & $23 \pm 1$ & $-58 \pm 9$ & $0.38 \pm 0.01$ \\
$1531 \pm 3$ & $5 \pm 2$ & $5 \pm 2$ & $-180 \pm 27$ & $0.28 \pm 0.03$ \\
$1565 \pm 2$ & $12 \pm 2$ & $7.6 \pm 0.9$ & $-70 \pm 13$ & $0.40 \pm 0.02$ \\
$1581 \pm 1$ & $5.6 \pm 0.8$ & $8 \pm 1$ & $-57 \pm 14$ & $0.38 \pm 0.02$ \\
$1599 \pm 2$ & $7 \pm 2$ & $7 \pm 1$ & $-150 \pm 13$ & $0.39 \pm 0.07$ \\
$1606 \pm 2$ & $4 \pm 2$ & $3.1 \pm 0.9$ & $104 \pm 10$ & $0.55 \pm 0.14$ \\
$1655.6 \pm 0.7$ & $5.4 \pm 0.6$ & $19.9 \pm 0.9$ & $-34 \pm 11$ & $0.40 \pm 0.01$ \\
\hline
\end{tabular}

a Background amplitude $\chi_{\mathrm{E} t \mathrm{~h}}^{(3) \mathrm{NR}}=9.16, \phi^{\mathrm{NR}}=0$, and $\rho^{\mathrm{NR}}=1 / 3$. Parameter scaling factor for Fig. 4: (a) 1.00; (b) 1.00; (c) 3.88 .

interference with the electronic susceptibility $\chi_{S_{1}}^{(3) \mathrm{E}}$. However, one needs a pronounced solvent band in the spectral range. In our case the ethanol line around $1455 \mathrm{~cm}^{-1}$ is too weak for this purpose.

Fit Program. The measured polarization CARS spectra as shown in Figs. 4 and 5 were fitted with a nonlinear least-squares procedure. The normalized CARS signal $I_{A} / I_{1}^{2} I_{2}$ is modeled by the function

$$
I_{A}(x)=\left(\chi^{(3)}(x)\right)\left(\chi^{(3)}(x)\right)^{*}=\left|\chi^{(3)}(x)\right|^{2}
$$

where

$$
\begin{aligned}
\chi^{(3)}(x)= & {\left[p^{\mathrm{NR}} \chi_{\mathrm{Eth}}^{(3) \mathrm{NR}}+\sum_{t} p_{t \mathrm{Eth}}^{\mathrm{R}} \frac{\chi_{t \mathrm{Eth}}^{(3) \mathrm{R}}}{\left(\Omega_{t \mathrm{Eth}}^{\mathrm{R}}-x\right) / \Gamma_{t \mathrm{Eth}}^{\mathrm{R}}-i}\right.} \\
& +p^{\mathrm{E}} \chi_{S_{1}}^{(3) \mathrm{E}} \exp \left(i \theta_{1}^{\mathrm{E}}\right) \\
& \left.+\sum_{s=0}^{1} \sum_{t} p_{s t}^{\mathrm{R}} \frac{\chi_{s t}^{(3) \mathrm{R}} \exp \left(i \theta_{s t}^{\mathrm{R}}\right)}{\left(\Omega_{s t}^{\mathrm{R}}-x\right) / \Gamma_{s t}^{\mathrm{R}}-i}\right] c
\end{aligned}
$$

where

$$
\left.\begin{array}{rl}
p^{\mathrm{NR}} & =\cos \phi \cos \nu-\rho^{\mathrm{NR}} \sin \phi \sin \nu \\
p_{t \mathrm{Eth}}^{\mathrm{R}} & =\cos \phi \cos \nu-\rho_{t \mathrm{Eth}}^{\mathrm{R}} \sin \phi \sin \nu \\
p^{\mathrm{E}} & =\cos \phi \cos \nu-\rho^{\mathrm{E}} \sin \phi \sin \nu \\
p_{s t}^{\mathrm{R}} & =\cos \phi \cos \nu-\rho_{s t}^{\mathrm{R}} \sin \phi \sin \nu
\end{array}\right\} \text { polarization }
$$

and $c$ is a scaling parameter.

In Eq. $15 \chi_{\mathrm{Eth}}^{(3) \mathrm{NR}}$ represents the nonresonant susceptibility arising from the solvent (ethanol) and $\chi_{S_{1}}^{(3) E}$ the pure electronic susceptibility of the $S_{1}$ state of rhodamine $6 \mathrm{G}$ with phase shift $\theta_{1}^{\mathrm{E}}$ with respect to the real $\chi_{\mathrm{Eth}}^{(3) \mathrm{NR}}$. The Raman resonances of both the $S_{0}$ and $S_{1}$ state of rhodamine 6G are given by the last term in Eq. 15, where $\chi_{s t}^{(3) \mathrm{R}}, \theta_{s t}^{\mathrm{R}}, \Omega_{s t}^{\mathrm{R}}$, and $\Gamma_{s t}^{\mathrm{R}}$ represent real amplitudes, phases, frequencies, and bandwidths (HWHM). The Raman resonances of the solvent ethanol are given in the second term in Eq. 15. The independent variable $x$ represents the frequency difference $\omega_{1}-\omega_{2}$. in the measured region $\left(\omega_{1}-\omega_{2}\right) / 2 \pi c=1450-1700 \mathrm{~cm}^{-1}$, the variation of $x$ is small in comparison to the electronic bandwidth $\Gamma_{n}^{\mathrm{E}} \approx$ $1000 \mathrm{~cm}^{-1}$ of the $S_{1} \rightarrow S_{n}$ transitions; therefore $\chi_{S_{1}}^{(3) \mathrm{E}}$ can

\begin{tabular}{|c|c|c|c|c|}
\hline$\Omega_{t}^{\mathrm{R}}\left(\mathrm{cm}^{-1}\right)$ & $\Gamma_{t}^{R}\left(\mathrm{~cm}^{-1}\right)$ & $\chi_{t}^{(3) \mathrm{R}}$ (a.u.) & $\theta_{t}^{\mathrm{R}}(\mathrm{deg})$ & $\begin{array}{c}\rho_{i}^{\mathrm{R}}= \\
\chi_{2112}^{(3) R} / \chi_{1111}^{(3) R}\end{array}$ \\
\hline $1463 \pm 3$ & $8 \pm 3$ & $4 \pm 1$ & $50 \pm 13$ & $0.33 \pm 0.01$ \\
\hline $1489 \pm 4$ & $11 \pm 5$ & $5 \pm 1$ & $95 \pm 14$ & $0.39 \pm 0.02$ \\
\hline $1515 \pm 3$ & $4 \pm 2$ & $3.4 \pm 0.6$ & $-60 \pm 30$ & $0.50 \pm 0.15$ \\
\hline $1531 \pm 1$ & $6.0 \pm 0.8$ & $10 \pm 1$ & $-150 \pm 8$ & $0.17 \pm 0.02$ \\
\hline $1547 \pm 3$ & $10 \pm 3$ & $6 \pm 1$ & $-136 \pm 13$ & $0.06 \pm 0.04$ \\
\hline $1565 \pm 9$ & $12 \pm 10$ & $2 \pm 1$ & $16 \pm 24$ & $0.65 \pm 0.10$ \\
\hline $1581 \pm 4$ & $10 \pm 4$ & $4 \pm 1$ & $-51 \pm 21$ & $0.62 \pm 0.09$ \\
\hline $1599 \pm 3$ & $15 \pm 5$ & $8 \pm 2$ & $159 \pm 9$ & $0.31 \pm 0.01$ \\
\hline $1606 \pm 2$ & $19 \pm 8$ & $5 \pm 1$ & $-71 \pm 22$ & $0.35 \pm 0.01$ \\
\hline $1618 \pm 20$ & $19 \pm 20$ & $1 \pm 2$ & $-42 \pm 40$ & $0.22 \pm 0.10$ \\
\hline $1655 \pm 3$ & $5 \pm 2$ & $4 \pm 1$ & $-74 \pm 36$ & $0.50 \pm 0.15$ \\
\hline
\end{tabular}
be taken as a real constant amplitude.

In Eq. 16 the polarization factors $p^{\mathrm{NR}}, p^{\mathrm{E}}$, and $p_{s t}^{\mathrm{R}}$ represent the projections of the CARS signal onto the analyzer; $\phi$ is the angle between the CARS probe polarizations $\vec{e}_{1}$ and $\vec{e}_{2}$; and $\nu$ is the angle between the analyzer
TABLE 1II. Vibrational data of rhodamine 6G with pre-excitation (Fig. 5).

a Background amplitude $\chi_{\mathrm{ELh}}^{(3) \mathrm{NR}}=9.16, \phi^{\mathrm{NR}}=0$ and $\rho^{\mathrm{NR}}=1 / 3$. Electronic amplitude $\chi_{s_{1}^{(3) \mathrm{E}}}=8.75, \theta_{1}^{\mathrm{E}}=-74.8^{\circ}$, and $\rho^{\mathrm{E}}=0.22$. Parameter scaling factor for Fig. 5: (a) 0.71; (b) 1.05 .

$\vec{e}_{A}$ and $\vec{e}_{1} \cdot \rho^{\mathrm{NR}}, \rho^{\mathrm{E}}$, and $\rho_{s t}^{\mathrm{R}}$ represent the depolarization ratios of the nonresonant background, pure electronic $S_{1}$ state, and Raman (vibration) resonant contributions, respectively.

The intensity is given by the squared modulus of the third-order susceptibility, which means that all terms in Eq. 15 interfere, giving rise to complex line shapes. The fit program enables us to extract all parameters in Eqs. 14-16 by minimizing

$$
\sum_{j} \sum_{i}\left[M_{j}\left(x_{i}\right)-I_{j}\left(x_{i}\right)\right]^{2}
$$

where $i$ notes the number of spectral points, and $j$ the different polarization conditions. $M_{j}\left(x_{i}\right)$ is the measured CARS intensity at spectral point $x_{i}$ under polarization condition $j$, and $I_{j}\left(x_{i}\right)$ the modeled intensity given in Eqs. 14 and 15. The fit procedure is as follows:

Ground-State Spectra without Pump $\chi_{S_{1}}^{(3) \mathrm{E}}=0$.

(a) The ground-state $S_{0}$ polarization CARS $\chi_{111}^{(3)}$ spectrum (with $\vec{e}_{A} \| \vec{e}_{1}$ ) is fitted with free parameters: $\Omega_{0 t}^{\mathrm{R}}$ and $\Gamma_{0 t}^{\mathrm{R}}, \chi_{0 t}^{\mathrm{R}}, \theta_{0 t}^{\mathrm{R}}$, and $\chi_{\mathrm{Eth}}^{(3) \mathrm{NR}}$. The polarization angles are set at the experimental values. The nonresonant depolarization ratio is assumed to be $\rho^{\mathrm{NR}}=1 / 3$.

(b) With the set of parameters obtained in (a) the three polarization CARS spectra $\left[\chi_{1111}^{(3)}, \chi_{2112}^{(3)}\left(\vec{e}_{A} \perp \vec{e}_{1}\right)\right.$, and background suppressed] are fitted, now with fixed parameters $\chi_{\mathrm{E} t h}^{(3 \mathrm{NR}}, \Omega_{0 t}^{\mathrm{R}}, \Gamma_{0 t}^{\mathrm{R}}, \chi_{0 t}^{\mathrm{R}}$, and $\theta_{0 t}^{\mathrm{R}}$ and free parameters $\rho_{s t}^{\mathrm{R}}$ and scaling parameters $c$ for the $\chi_{2112}^{(3)}$ and background suppressed spectra.

\section{CARS Spectra with Pump.}

(c) Starting off with the parameter set obtained in (b) $\chi_{S_{1}}^{(3) \mathrm{E}}$ is taken to be $\chi_{S_{1}}^{(3) \mathrm{E}}=\left|\vec{P}^{\mathrm{E}} / \vec{P}^{\mathrm{NR}}\right| \chi^{(3) \mathrm{NR}}=$ $0.956 \chi^{(3) \mathrm{NR}}$, and the phase $\theta_{1}^{\mathrm{E}}$ is set at $-75^{\circ}$, as estimated from the ellipsometry measurements (see above). Both parameters $\chi_{S_{1}}^{(3)}$ and $\theta_{1}^{E}$ are fixed. The pumped $\chi_{1111}^{(3)}$ spectrum $\left(\vec{e}_{A} \| \vec{e}_{1}\right)$ is fitted with additional parameters $\Omega_{1 t}^{\mathrm{R}}, \Gamma_{1 t}^{\mathrm{R}}, \chi_{1 t}^{\mathrm{R}}$, and $\theta_{1 t}^{\mathrm{R}}$ for the $S_{1}$ vibrations and free amplitudes $\chi_{0 t}^{\mathrm{R}}$ and phases $\theta_{0 t}^{\mathrm{R}}$ of the $S_{0}$ vibrations to account for depletions of the ground state $S_{0}$ and the change of line shapes due to interference with $\chi_{S_{1}}^{(3) \mathrm{E}}$ and $\theta_{1}^{\mathrm{E}}$. 
(d) The pumped $\chi_{2112}^{(3)}$ and $\chi_{1111}^{(3)}$ spectra are fitted with the parameter set obtained in (c) with depolarization ratios $\rho_{0 t}^{\mathrm{R}}$ and $\rho_{1 t}^{\mathrm{R}}$ as free parameters, while the others are fixed. $\rho^{\mathrm{E}}$ is fixed at the theoretical value 0.22 . Free scaling parameters of both $\chi_{1111}^{(3)}$ and $\chi_{2112}^{(3)}$ spectra in (c) and (d) are used to account for the absolute change in intensity, due to weak thermal lens scattering of the CARS probe beams.

With the use of this procedure the best fit parameters listed in Table II (without pump) and Table III (with pump) were obtained.

\section{DISCUSSION}

Depolarization Ratios. The CARS spectra in Fig. 4 show pronounced Raman bands at 1516, 1565, 1581, 1599, and $1656 \mathrm{~cm}^{-1}$ assigned to ground-state rhodamine $6 \mathrm{G} .{ }^{10}$ The fits on these spectra are of good quality if bands at 1531 and $1606 \mathrm{~cm}^{-1}$ are introduced, which have been assigned to the excited singlet $S_{1}$ state of rhodamine $6 \mathrm{G}^{10}$ This means that although $E_{1}<E_{2}$ the excited state $S_{1}$ is populated to a small extent.

With pre-excitation, dramatic changes in the spectra are observed. Excitation of rhodamine $6 \mathrm{G}$ molecules induces a high degree of population of the excited $S_{1}$ state, giving strong bands at 1531 and $1606 \mathrm{~cm}^{-1}$, which are enhanced due to the higher electronic states $S_{3}$ and $S_{4}$ (see Fig. 5). The ground state is highly depleted, which can be seen from the diminished intensities of the goundstate bands at 1517 and $1655 \mathrm{~cm}^{-1}$. The $6.7 \times$ decrease in intensities corresponds to an excited-state population of $85 \%$. This result agrees well with estimated excitedstate concentrations of rhodamine $6 \mathrm{G}$ in dependence on the incident photon flux. ${ }^{20}$ In addition a high pedestal is seen that results from a high $\chi_{S_{1}}^{(3) E}$ value (Table III), which is comparable to the value of $\chi_{\mathrm{Eth}}^{(3) N R}$. Taking the concentration difference into account (viz., $2.1 \cdot 10^{-4} \mathrm{M}$ and 17.15 $\mathrm{M})$, we see that excited rhodamine $6 \mathrm{G}$ has a very large molecular hyperpolarizability. Furthermore, interference of $\chi_{\mathrm{Eth}}^{(3) \mathrm{NR}}, \chi_{0 t}^{(3) \mathrm{R}}$, and $\chi_{1 t}^{(3) \mathrm{R}}$ with the complex electronic susceptibility $\chi_{S_{1}}^{(3) E}$ gives different band shapes in comparison to the ones in Fig. 4. Comparing the $\chi_{1111}^{(3) R}$ and $\chi_{2112}^{(3) \mathrm{R}}$ components in Figs. 4 and 5 , we see that, upon excitation, differences in depolarization ratios are observed.

The depolarization ratios $\rho_{0 t}^{\mathrm{R}}$ of the ground-state bands, as estimated from the fits (see Table II), have values $(0.38 \pm 0.02 \ldots 0.40 \pm 0.02)$ close to the nonresonant depolarization ratio $\rho^{\mathrm{NR}}=1 / 3$. A depolarization ratio of $1 / 3$ is generally expected for vibrations that are (pre-) resonantly enhanced by a single electronic transition with a fixed molecular direction. ${ }^{21}$ For symmetric vibrations the isotropic and anisotropic Raman tensor elements then reduce to $\bar{\alpha}=1 / 3 \bar{\alpha}_{x x}, \bar{\gamma}_{s}=\bar{\alpha}_{x x}$, and $\bar{\gamma}_{a}=0$ (with $x$ the direction along the transition moment), which yields a depolarization ratio:

$$
\rho^{\mathrm{R}}=\frac{-5 \bar{\gamma}_{a}^{2}+3 \bar{\gamma}_{s}^{2}}{45 \bar{\alpha}^{2}+4 \bar{\gamma}_{s}^{2}}=1 / 3
$$

Deviations of the depolarization ratio from $1 / 3$ imply either that more electronic transitions with different directions of the transition moments in the molecule make a contribution or that the vibrations have antisymmetric character. However, the symmetry of the rhodamine $6 \mathrm{G}$ molecule, disregarding the side groups, is $C_{2 V}$, leading to $A_{1}$ and $B_{2}$ vibrational symmetries. In the molecular structure (see Fig. 3A), the orientations of the dipole moments of the one-photon-allowed $S_{0}-S_{1}$ and $S_{0}-S_{n}$ transitions are along the $y$-axis of the rhodamine $6 \mathrm{G}$ molecule, whereas the dipole moment of the excited-state transition $S_{1}-S_{n}^{\prime}$ is perpendicular to this direction (z-axis). The depolarization ratios close to $1 / 3$ are thus mainly caused by the strong transition dipole moment of the $S_{1}$ band along the $y$-axis of the rhodamine $6 \mathrm{G}$ molecule. The small deviation from 1/3 may arise from the influence of the higher electronic transitions $S_{0}-S_{2}, \ldots, S_{n}$.

It is remarkable that the depolarization ratios obtained from the fit on the spectra with and without pump are different. The change in depolarization ratios may be caused by several factors. First, as discussed above, anisotropy in the distribution of induced dipole moments in the excited-and ground-state molecules imposes different relationships between the third-order susceptibility components $\chi_{i j k l}^{(3)}$, yielding different depolarization ratios. Second, the CARS process in the excited $S_{1}$ state is enhanced by the different electronic transition $S_{1}-S_{n}^{\prime}$ with the orientation of the transition dipole moment perpendicular ${ }^{12}$ to those of the ground state $S_{0}-S_{n}$ (see Fig. 3). The CARS process in the ground state $S_{0}$ is enhanced by electronic transitions $S_{0}-S_{n}$, thereby enhancing vibrations differently. Third, the structure of the rhodamine $6 \mathrm{G}$ molecule may have altered upon excitation, which could lead to a change in the molecular symmetry and thus to a different symmetry of the vibrations in the excited state. Since depolarization ratios are related to vibrational symmetry, changes in molecular symmetry may alter depolarization ratios.

Estimated depolarization ratios of the Raman bands that follow from Tables II and III of the spectra with pump (filled circles) and without pump (open circles) are depicted in Fig. 11. The ground-state rhodamine $6 \mathrm{G}$ vibrations at $1515,1565,1581,1599$, and $1655 \mathrm{~cm}^{-1}$ have depolarization ratios in the range $\rho_{0 t}^{\mathrm{R}}=(0.38 \pm 0.02) \ldots$ $(0.40 \pm 0.02)$, while for the spectra obtained with pump the depolarization ratios are in the range $\rho_{0 t}^{\mathrm{R}}=(0.5 \pm$ $0.15) \ldots(0.65 \pm 0.10)$ [except for the $1599-\mathrm{cm}^{-1}$ band with $\left.\rho_{0 t}^{\mathrm{R}}=(0.31 \pm 0.01)\right]$. The vibrations in the pumped spectra at $1531,1547,1606$, and $1618 \mathrm{~cm}^{-1}$ have $\rho_{1 t}^{\mathrm{R}}=$ $(0.06 \pm 0.04) \ldots(0.22 \pm 0.10)$. The $1599-\mathrm{cm}^{-1}$ band shows excited-state behavior, since its amplitude increases and its depolarization ratio decreases in the pumped spectra.

The larger errors in the estimated depolarization ratios of the ground-state bands, in comparison with that of the excited-state bands in the spectrum with pump, are due to the decreased amplitudes of the former (depletion of the ground state) and increased amplitudes of the latter (excited-state population).

From the estimated depolarization ratio for the ground state $(0.5 \ldots 0.65)$, which agrees well with the predicted value 0.54 , we conclude that these effects are small during the pulse. The change of the depolarization ratios of ground-state vibrations $\Delta \rho_{0 t}^{\mathrm{R}}=\rho_{0 t}^{\mathrm{R}}$ (with pump) $-\rho_{0 t}^{\mathrm{R}}$ (without pump) $=(0.10 \pm 0.16) \ldots(0.25 \pm 0.12)$ is in agreement with a calculated change of $\Delta \rho_{0 t}^{\mathrm{R}}=0.21$ for a band with $\rho_{0 t}^{\mathrm{R}}=1 / 3$ and excited-state fraction $\mathrm{g}_{1}=0.85$ 
where we have used Eq. A7 of Appendix A in calculating the depolarization ratio $\rho_{\text {ANIS }}^{S_{0}}=0.54$ of the anisotropic ground state.

Since calculated depolarization ratios of the groundstate bands in the spectra with pump agree with the predicted value 0.54 , we may state that the assumption of parallel transition moments for the CARS process holds. From the orientation of the dipole moment of the transition $S_{0}-S_{n}$, this means that these lie along the y-axis in the molecule. This observation justifies the choice made in the second part of the Results section. The estimated differences in the depolarization ratios of excited vibrations with the predicted value $\rho^{\mathrm{E}}=(0.22 \pm 0.07)$ (see the fourth part of the Results section) may be explained from the contributions of different electronic enhancements (i.e., $S_{1}-S^{\prime}{ }_{n}$ enhancements) or nonparallel transition moments in the excited molecule. The latter would be the result of a changed excited molecular geometry. The former also affects the vibrational phases. This observation may be seen from a more detailed expression of the third-order susceptibility

$$
\chi_{\mathrm{A} 112}^{(3) \mathrm{R}} \sim \sum_{m, n} \rho^{(0,1)} \frac{\left\langle\left(\vec{\mu}_{\alpha} \cdot \vec{e}_{A}\right)\left(\vec{\mu}_{\beta} \cdot \vec{e}_{1}\right)\left(\vec{\mu}_{\gamma} \cdot \vec{e}_{1}\right)\left(\vec{\mu}_{\delta} \cdot \vec{e}_{2}\right)\right\rangle_{\mathrm{oa}}}{\left[\left(\Omega_{m}-\omega_{1}-i \Gamma_{m}\right)\left(\Omega_{n}-\omega_{a}-i \Gamma_{n}\right)\right.}
$$

where the first two factors in the denominator represent the electronic resonance enhancement at the CARS probe frequency $\omega_{1}$ and the CARS signal frequency $\omega_{a}=2 \omega_{1}$ $\omega_{2}$, with $\Omega_{m}, \Omega_{n}$ and $\Gamma_{m}, \Gamma_{n}$ electronic transition frequencies and bandwidths, respectively. The third factor is the vibrational resonant factor. The inner products $(\vec{\mu} \cdot \vec{e})$ are projections of the molecular dipole moments onto the polarization vectors of the CARS probe fields and signal analyzer. $\rho^{(0,1)}$ denotes the occupation of the ground state $S_{0}$ and excited state $S_{1}$. In the summation, $m$ and $n$ run over all the excited electronic states.

From Eq. 19 it is clear that each susceptibility component, in general, is built up from a number of electronic enhancements weighted by the orientational averaged quadruple inner product of dipole moments and polarization vectors. Since we use the shorthand notation

$$
\chi_{t}^{(3) \mathrm{R}}=\chi_{t}^{\mathrm{R}} \exp \left(i \theta_{t}^{\mathrm{R}}\right) /\left(\delta_{t}-i \Gamma_{t}\right)
$$

it is seen from comparing Eqs. 19 and 20 that not only does the amplitude carry information on the enhancements but the phase also depends on it if more than one electronic state contributes. Since the depolarization ratio $\rho^{\mathrm{R}}$ is the ratio of two $\chi^{(3)}$ components, each consisting of a sum given in Eq. 19 with specific weights $\left\langle\left(\vec{\mu}_{\alpha}\right.\right.$. $\left.\left.\vec{e}_{A}\right)\left(\vec{\mu}_{\beta} \cdot \vec{e}_{1}\right)\left(\vec{\mu}_{\gamma} \cdot \vec{e}_{1}\right)\left(\vec{\mu}_{\delta} \cdot \vec{e}_{2}\right)\right\rangle_{\text {oa }}$, this case in general results in different depolarization ratios for different electronic manifolds due to possible variation in transition dipole moment orientations. Furthermore, each vibration may couple to vibrations of excited electronic states (vibronic coupling), not denoted in Eq. 20, which may also affect the differences in the phases of different vibrations of the same electronic state.

The origin of the depolarization ratio changes observed could be based solely on induced anisotropy in both excited and ground state. However, structural changes in the excited state may have occurred as well, and, as dis-

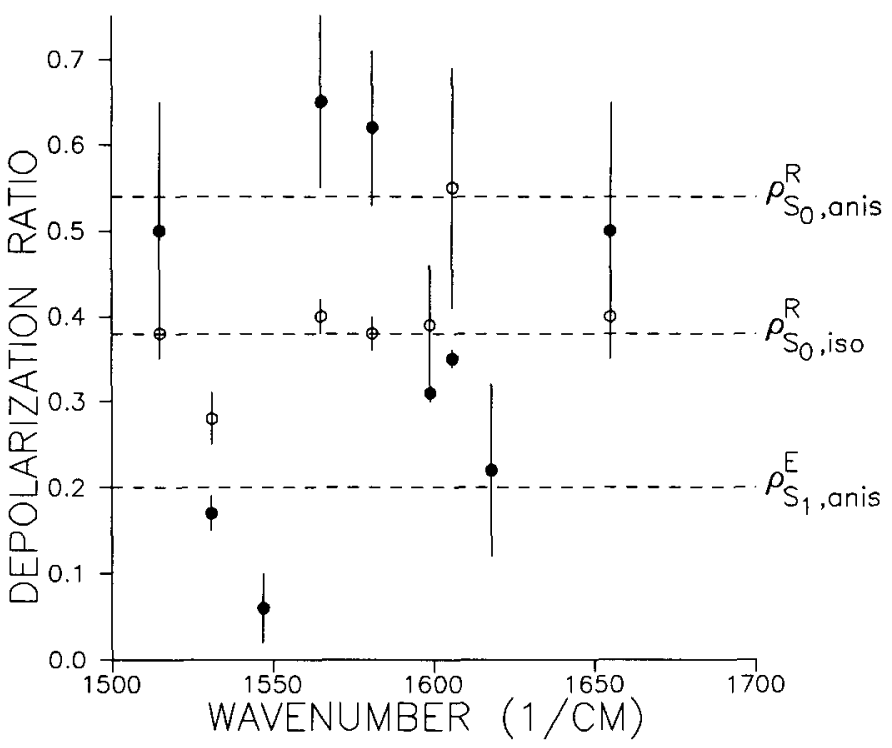

FIG. 11. Estimated vibrational depolarization ratios with (filled circles) and without (open circles) pumping. The horizontal dashed lines indicate isotropic ground-state value $\rho_{S_{0} \text {, Iso }}^{\mathrm{R}}=0.38$ (without pump), anisotropic ground-state $\rho_{S_{0} \text { ANIS }}^{\mathrm{R}}=0.54$, and excited-state $\rho_{S_{1}, \text { ANIS }}^{\mathrm{R}}=0.22$ (with pump).

cussed above, different electronic enhancements may also contribute to depolarization ratio changes. At this point it seems difficult to distinguish between these effects. Knowledge of electronic excited-state molecular geometry is necessary to obtain true molecular depolarization ratios in the excited state.

Vibrational Phases. The vibrational phases obtained from the fits on the spectra (see Tables II and III) are determined with respect to a real nonresonant contribution $\chi_{\mathrm{Eth}}^{(3) \mathrm{NR}}$ of ethanol with phase $\phi^{\mathrm{NR}}=0$. The phase of the pure electronic contribution was fixed at $\theta_{1}^{\mathrm{E}}=-75^{\circ}$ as determined in the fifth part of the Results section. To visualize the results, note the nonresonant background $\chi_{\mathrm{Eth}}^{(3) \mathrm{NR}}$, pure electronic $\chi_{S_{1}}^{(3) \mathrm{E}}$, and vibration resonant $\chi_{s t}^{(3) \mathrm{R}}$ susceptibilities that are depicted in the complex plane as vectors (i.e., amplitude and phase), shown in Fig. 12. The nonresonant susceptibility of ethanol $\chi_{\mathrm{Eth}}^{(3) \mathrm{NR}}$ is a real constant. The pure electronic contribution $\chi_{S_{1}}^{(3) \mathrm{E}} \exp \left(i \theta_{1}^{\mathrm{E}}\right)$ is a vector lying at an angle $\theta_{1}^{\mathrm{E}}$ away from the positive Rex axis. The vibrational contributions $\chi_{s t}^{(3) \mathrm{R}} \exp \left(i \theta_{s t}^{\mathrm{R}}\right) /$ $\left(\delta_{s t}-i \Gamma_{s t}\right)$ are shown at resonance, (i.e., $\delta_{s t}=0$ ), the vectors starting from the resultant of $\chi_{\mathrm{Eth}}^{(3) \mathrm{NR}}+$ $\chi_{S_{1}}^{(3) \mathrm{E}} \exp \left(i \theta_{1}^{\mathrm{E}}\right)$ and rotated by $\theta_{s t}^{\mathrm{R}}$ counter-clockwise from the positive imaginary axis $\operatorname{Im} \chi$. A zero phase $\theta_{s t}^{\mathrm{R}}$ would belong to vibrations of ethanol since these are far off electron resonance. The ethanol band at $1455 \mathrm{~cm}^{-1}$ was fitted with a free phase which resulted in $21^{\circ}$; however, this band lies at the edge of the spectrum, which makes analysis difficult and may explain the deviation from zero. The ground-state vibrations of rhodamine $6 \mathrm{G}$ have phases different from zero, due to the electronic resonance enhancement of the $S_{1}$ and $S_{2}$ transitions.

Comparing Figs. 12A and 12B it is immediately seen that, upon pumping, most vibrational amplitudes decrease due to ground-state depletion, and that the total background, represented by $\chi_{\mathrm{Eth}}^{(3) \mathrm{NR}}$ and $\chi_{S_{1}}^{(3) \mathrm{E}}$, is larger (and complex), corresponding to the higher peak in Fig. 5. 

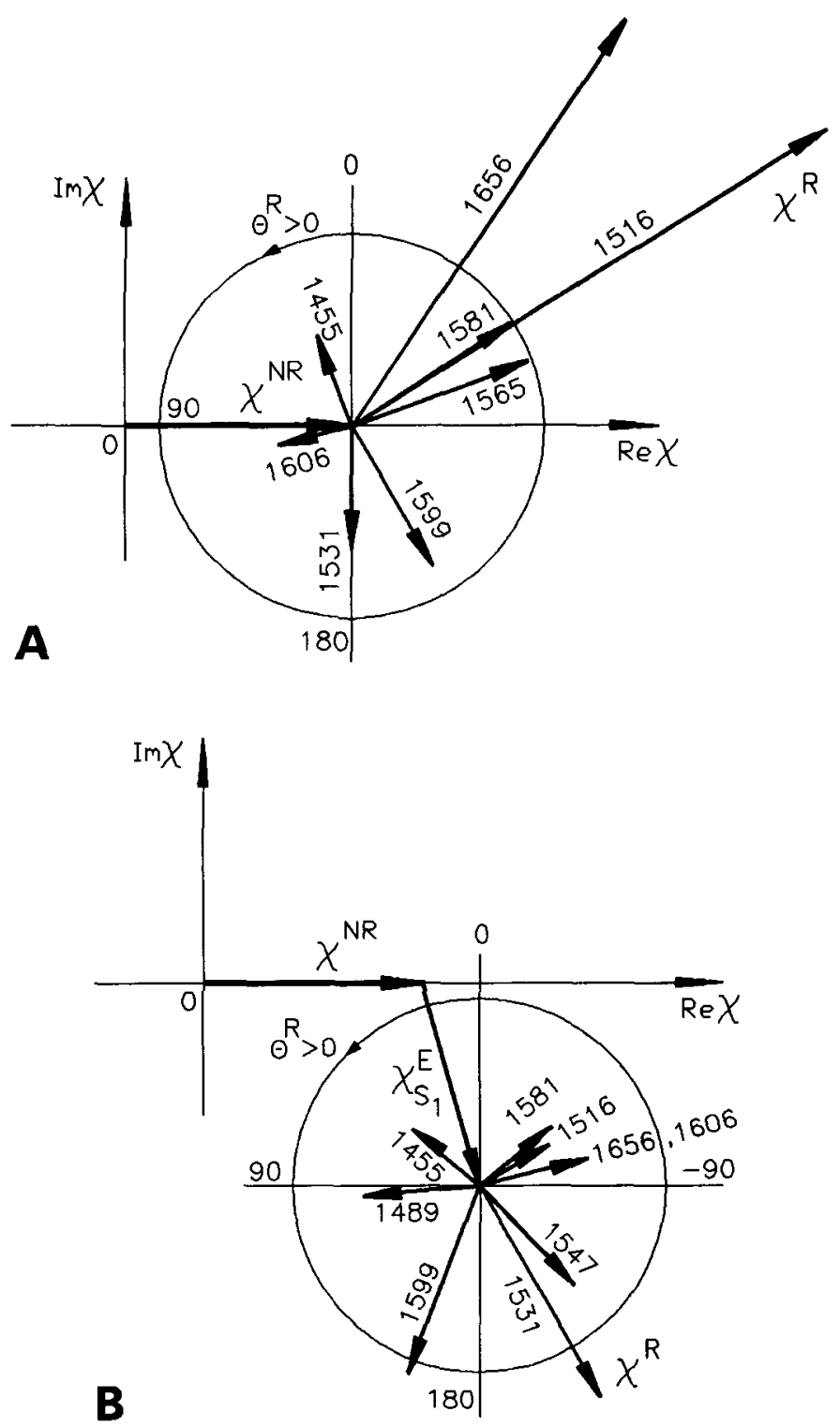

FIG. 12. Susceptibility contributions represented in the complex plane of nonresonant background $\chi_{\mathrm{Eh}}^{(3) \mathrm{NR}}$, pure electronic $\chi_{\mathrm{S1}}^{(3) \mathrm{E}} \exp \left(i \theta_{1}^{\mathrm{E}}\right)$, and vibrational resonances $\chi_{s t}^{(3) \mathrm{R}} \exp \left(i \theta_{s t}^{\mathrm{R}}\right) /\left(-i \Gamma_{s t}^{\mathrm{R}}\right)$ : (A) Ground state (without pump), and (B) excited state (with pump). The phases are obtained with respect to a real background phase $\phi^{\mathrm{NR}}=0$.

From Fig. 12A it is clear that the most pronounced ground-state bands at 1516,1581 , and $1656 \mathrm{~cm}^{-1}$ have phases in the range $-34^{\circ}$ to $-70^{\circ}$, different from zero due to resonance enhancement, as discussed above. In the spectrum with pump (Fig. 12B) no prominent phase changes are observed for these bands. This result indicates that these bands exist only in the ground-state conformation. The bands at 1531,1547 , and $1599 \mathrm{~cm}^{-1}$ have phases in the -150 to $-200^{\circ}$ range in the spectrum with and without pump. This range differs from that of the ground-state bands due to the different enhancements (position of the CARS probes in the excited-state absorption band $S_{1}-S_{n}^{\prime}$ ). This observation confirms that these bands really belong to the excited state. The bands at 1606 and $1618 \mathrm{~cm}^{-1}$ also show excited-state character as judged by their depolarization ratio changes in the pumped and unpumped spectra. Their different phases, in comparison to those of the other excited-state bands, may be due to different coupling with the electronic states $S_{n}$ or $S_{n}^{\prime}$.

Estimation of the Hyperpolarizabilities $\gamma_{S_{0}}^{E}$ and $\gamma_{S_{1}}^{E}$ of Rhodamine 6G. With the value $\left|\vec{P}^{\mathrm{E}}\right|=0.956\left|\vec{P}^{\mathrm{NR}}\right|$ as obtained from the coherent ellipsometry measurements in the fourth part of the Results section, we can estimate the value of the hyperpolarizability $\gamma_{S_{1}}^{\mathrm{E}_{1}}$ of the excited $S_{1}$ state of rhodamine $6 \mathrm{G}$. The photon flux given by $\phi=$ $E_{\mathrm{pu}} /\left(A_{f} \tau_{\mathrm{p}} E_{\mathrm{ph}}\right)=1.5 \cdot 10^{27}$ (photons $/ \mathrm{cm}^{2} \mathrm{~s}$ ) in the peak. Here the energy in the pulse $E_{\mathrm{pu}}=1 \mathrm{~mJ}$, the energy of the photon (at $\lambda=532 \mathrm{~nm}$ ) $E_{\mathrm{ph}}=3.74 \cdot 10^{-19} \mathrm{~J}$, the focal area $A_{\mathrm{f}}=4.91 \cdot 10^{-4} \mathrm{~cm}^{2}$, and the pulse duration $\tau_{\mathrm{p}}=8 \mathrm{~ns}$. With this photon flux it follows from Ref. 22 that the concentration of excited molecules is $N_{1}=1.25 \cdot 10^{17} \mathrm{~cm}^{-3}$, which is $83 \%$ of the ground-state concentration without pump. This high value of the excited-state concentration is in accordance with the strong $(6.7 \times)$ depletion of the ground-state $1516-\mathrm{cm}^{-1}$ vibration $(1-1 / 6.7=0.85)$.

With the value of the nonresonant susceptibility of ethanol at $\chi_{\mathrm{Eth}}^{(3) \mathrm{NR}}=3.65 \cdot 10^{-14} \mathrm{esu}^{23}$ and the molarity of pure ethanol at $17.5 \mathrm{M}$, it follows for the molecular hyperpolarizability $\gamma_{\mathrm{Eth}}^{\mathrm{NR}}=3.55 \cdot 10^{-39}$ esu. With this value the molecular hyperpolarizability for excited state of rhodamine $6 \mathrm{G}$ is given by

$$
\gamma_{S_{1}}^{\mathrm{E}}=0.956 \gamma_{\mathrm{Eth}}^{\mathrm{NR}}\left(17.15 / N_{1}\right)=27.3 \cdot 10^{-35} \mathrm{esu} \text {. }
$$

The value of the ground-state hyperpolarizability $\gamma_{S_{0}}^{\mathrm{E}}$ is estimated from $\chi_{S_{0}}^{(3) E}=0.158 \chi_{E \text { Eh }}^{(3) N R}$ as obtained from Table $\mathrm{I}$, and the number of molecules $N_{0}=1.5 \cdot 10^{17} \mathrm{~cm}^{-3}$, which yields:

$$
\gamma_{S_{0}}^{\mathrm{E}}=0.158 \gamma_{\mathrm{Eth}}^{\mathrm{NR}}\left(17.15 / N_{0}\right)=3.8 \cdot 10^{-35} \text { esu. }
$$

This value is close to that reported by Lau and Pfeiffer ${ }^{24}$ $\left(\gamma_{S_{0}}^{\mathrm{E}}=4 \cdot 10^{-35} \mathrm{esu}\right)$, although their value corresponds to excitation at $\lambda=570 \mathrm{~nm}$. We would thus expect a larger value in the case of exact resonant excitation. The hyperpolarizability $\gamma_{S_{1}}^{\mathrm{E}}$ in the excited state is more than 7 times larger than the ground-state hyperpolarizability $\gamma_{S_{0}}^{\mathrm{E}}$, which may be due to the changed dipole moment direction in the $S_{1}-S_{n}^{\prime}$ transition (from along the y-axis to the z-axis). Assuming a fifth power-length dependence of the molecular hyperpolarizability ${ }^{25}$ this means a change in the charge separation of the molecule in the excited state of $50 \%$. This response can be due to the electronic enhancement or a changed electron distribution (delocalization).

\section{CONCLUSION}

Resonance polarization CARS spectra of the electronic ground state and excited singlet $S_{1}$ state of rhodamine 6G in ethanol were obtained with the pump-probe technique. Upon varying the polarization orientation of the pump beam, we measured different excited-state spectra due to the optically induced anisotropy of the excited state. From concentration-dependent measurements it was shown that the electronic contribution $\chi_{S_{0}}^{(3) E}$ of groundstate rhodamine $6 \mathrm{G}$ was small in comparison to the nonresonant ethanol contribution. The influence of the electronic susceptibility $\chi_{S_{1}}^{(3) \mathrm{E}}$ of the excited state with respect to the nonresonant susceptibility of ethanol as examined 
by coherent ellipsometry was characterized by the ratio $\left|\vec{P}^{\mathrm{E}} / \vec{P}^{\mathrm{NR}}\right|=0.956$ and phase difference $\theta_{1}^{\mathrm{E}}=-74.8^{\circ}$.

The changes of vibrational depolarization ratios differ for the spectra taken with and without pumping, which may originate from the optically induced anisotropy in both ground and excited state due to photoselection and/ or molecular aligning by the pump field, different molecular structure (symmetry) in the excited state, and different enhancement mechanisms from various electronic transitions from the excited state. The effects of changed molecular structure and/or symmetry and different enhancement mechanisms cannot be determined without making assumptions about, for example, the structure of the excited molecule. The observed changes in depolarization ratios upon pumping could be explained by induced anisotropy under the assumption that the dipole moments of the CARS process are all parallel to the dipole moment of the $S_{1}-S^{\prime}{ }_{n}$ transition. Deviations from these calculated values would then indicate changed molecular structure/symmetry and/or changed enhancements of the CARS process.

The differences in the vibrational phases reflect different enhancements and vibronic coupling for the CARS process in the ground state (via $S_{0}-S_{1,2}$ ) and excited singlet state (via $S_{1}-S_{n}{ }_{n}$ ).

The hyperpolarizabilities of the ground state and excited singlet state of rhodamine $6 \mathrm{G}$ were estimated to be $\gamma_{S_{0}}^{\mathrm{E}}=3.8 \cdot 10^{-35}$ esu and $\gamma_{S_{1}}^{\mathrm{E}}=27.3 \cdot 10^{-35}$ esu, respectively.

\section{ACKOWLEDGMENTS}

The authors wish to thank Prof. Dr. B. Bölger and Dr. V. F. Kamalov for valuable discussions.

1. S. A. J. Druet and J.-P. E. Taran, in Progress in Quantum Electronics, T. S. Moss and S. Stenholm, Eds. (Pergamon Press, New York, 1981), Vol. 7.

2. R. Brakel and F. W. Schneider, in Advances in Nonlinear Spectroscopy, R. J. H. Clark and R. E. Hester, Eds. (John Wiley, Chichester, 1988), Vol. 15, p. 149.

3. S. A. Payne and R. M. Hochstrasser, Optics 11, 285 (1986).

4. H. Hamaguchi, Chem. Phys. Lett. 126, 185 (1986).

5. V. F. Kamalov, N. I. Koroteev, B. N. Toleutaev, A. P. Shkurinov, and U. Stamm, J. Phys. Chem. 94, 5645 (1989).

6. W. Werncke, T. J. Tschol, H.-J. Weigmann, M. Pfeiffer, and A. Lau, J. Raman Spectrosc. 18, 323 (1987).

7. A. Lau, R. König, and M. Pfeiffer, Optics Commun. 32, 75 (1980).

8. V. F. Kamalov, N. I. Koroteev, A. P. Shkurinov, and B. N. Toleutaev, J. Mol. Struct. 217, 19 (1990).

9. H. Hamaguchi, in Advances in Infrared and Raman Spectroscopy, R. J. H. Clark and R. F. Hester, Eds. (John Wiley, Chichester, 1985), Vol. 12, p. 273.

10. M. Fickenscher, H.-G. Purucker, and A. Lauberau, Appl. Phys. Photophys. Chem. B 51, 207 (1990).

11. G. Dolan and C. R. Goldschmidt, Chem. Phys. Lett. 39, 320 (1976).

12. A. Penzkopfer and J. Wiedmann, Opt. Commun. 35, 81 (1980).

13. H. Takahashi, S. Matsubara, S. Hirukawa, and S. Nagata, in TimeResolved and Transient Spectroscopy, Proc. IX ICORS, M. Tsuboi, Ed. (Organizing Committee for the IXth International Conference on Raman Spectroscopy, Tokyo, Japan, 1984), p. 320.

14. R. Igarashi, Y. Adachi, and S. Maeda, J. Chem. Phys. 72, 4308 (1980).

15. T. A. H. M. Scholten, Ph.D. Thesis, University of Twente, The Netherlands (1989).

16. T. A. H. M. Scholten, G. W. Lucassen, F. F. M. de Mul, and J. Greve, Appl. Opt. 27, 3225 (1988).

17. A. Savitzky and M. J. E. Golay, Anal. Chem. 36, 1627 (1964).

18. B. N. Toleutaev, Ph.D. Thesis, Moscow State University, Moscow, Russia (1989).
19. M. Pfeiffer, A. Lau, and W. Werncke, J. Raman Spectrosc. 15, 20 (1984).

20. S. A. Akhmanov, A. F. Bunkin, S. Gt. Ivanov, and N. I. Koroteev, Sov. Phys. JETP 47, 667 (1978).

21. J. Behringer, in Raman Spectroscopy, H. A. Szymanski, Ed. (Plenum Press, New York, 1967), p. 205.

22. V. P. Klochkov, E. B. Verkhovskii, and V. L. Bogdanov, Opt. Spectrosc. 70, $322(1991)$.

23. G. R. Meredith, B. Buchgalter, and C. Hanzlik, J. Chem. Phys. 78, 1533 (1983).

24. A. Lau and M. Pfeiffer, XI European CARS Workshop, Florence, Italy (1992).

25. C. Flytzanis, in Quantum Electronics: A Treatise, H. Rabin and C. Tang, Eds. (Academic Press, New York, 1975), Vol. 1, p. 137.

\section{APPENDIX A: RELATIONS BETWEEN SUSCEPTIBILITY COMPONENTS IN THE CASE OF INDUCED ANISOTROPY}

To derive relations between the macroscopic susceptibility $\chi_{i j k l}^{(3) \mathrm{E}}$ components in the case of an optically induced anisotropy in the medium, one needs to average the microscopic susceptibility $\chi_{i j k l}^{(3,3 \mathrm{~mol} E}$ (or hyperpolarizability $\left.\gamma_{i j k l}^{(3) E}\right)$ components over all molecular orientations. Taking into account the anisotropic distribution functions in the excited state (Eq. 9 of the text)

$$
f_{S_{1}}(\theta)=N_{1} \cos ^{2} \theta
$$

and of the ground state

$$
f_{S_{0}}(\theta)=N_{0}-N_{1} \cos ^{2} \theta=N_{0}\left(1-g_{1} \cos ^{2} \theta\right)
$$

where $N_{0}$ and $N_{1}$ denote the ground-state and excitedstate concentration, $g_{1}=N_{1} / N_{0}$ represents the excited fraction, and $\theta$ is the angle between the pump beam polarization orientation and the transition dipole in the molecule, it follows

$$
\chi_{i j k l}^{(3) \mathrm{E}}=\left\langle\chi_{i j k l}^{(3 \mathrm{~mol}) \mathrm{E}}\right\rangle_{\mathrm{oa}}=L \tilde{\gamma}_{\alpha \beta \gamma \delta}^{(3) \mathrm{E}}\left\langle R_{i \alpha} R_{j \beta} R_{k \gamma} R_{l \delta} f_{\mathrm{S}_{0,1}}(\theta)\right\rangle_{\mathrm{oa}}
$$

where $\langle\ldots\rangle_{\text {oa }}$ denotes the orientational average, $L$ is a local-field correction factor given by $L=\left(\epsilon\left(\omega_{1}\right)+2\right)^{2}\left(\epsilon\left(\omega_{2}\right)\right.$ $+2)\left(\epsilon\left(\omega_{3}\right)+2\right) / 81$, with $\epsilon\left(\omega_{i}\right)$ the dielectric constant of the medium, and $\tilde{\gamma}_{\alpha \beta \gamma \delta}^{(3) E}$ the hyperpolarizability where the quadruple inner product of the molecular transition dipoles $\left(\vec{\mu}_{\alpha}, \vec{\mu}_{\beta}, \vec{\mu}_{\gamma}, \vec{\mu}_{\hat{\sigma}}\right)$ and the electric field polarization vectors $\left(\vec{e}_{i}, \vec{e}_{j}, \vec{e}_{k}, \vec{e}_{l}\right)$ denoted by $R_{i \alpha} R_{j \beta} R_{k \gamma} R_{l \delta}$ is factored out. Here $\alpha, \beta, \gamma$, and $\delta$ refer to coordinate axes in the molecular frame, whereas $i, j, k$, and $l$ refer to axes in the laboratory frame. the averaging can be written:

$$
\begin{aligned}
& \left\langle R_{i \alpha} R_{j \beta} R_{k \gamma} R_{l \delta} f_{S_{0,1}}(\theta)\right\rangle_{\text {oa }} \\
& =\frac{1}{8 \pi^{2}} \int_{\nu=0}^{2 \pi} \mathrm{d} \psi \int_{\theta=0}^{\pi} \mathrm{d} \theta \int_{\varphi=0}^{2 \pi} \mathrm{d} \varphi\left(\vec{\mu}_{\alpha} \cdot \vec{e}_{i}\right)\left(\vec{\mu}_{\beta} \cdot \vec{e}_{j}\right)\left(\vec{\mu}_{\gamma} \cdot \vec{e}_{k}\right) \\
& \times\left(\vec{\mu}_{\delta} \cdot \vec{e}_{l}\right) f_{S_{0,1}}(\theta) \sin \theta .
\end{aligned}
$$

The transformation of the molecular fixed axes to the laboratory axes is performed through the Euler angles $(\phi, \theta, \psi)$.

Since the photoselection leaves a distribution of the induced dipole moments with an inversion center, only 6 of the 21 nonzero components of the cubic susceptibility tensor $\chi_{i j k l}^{(3) E}$ are independent. Assuming parallel dipole moments in the molecule (i.e., corresponding to a 
nondegenerate fixed direction of the electronic transition in the molecular frame and a single nonzero factor $\tilde{\gamma}_{\alpha \alpha \alpha \alpha}^{(3) \mathrm{E}}=\tilde{\gamma}_{0} \neq 0$ ) and applying the anisotropic distribution function (A2) for $g_{1}=1$, we see the result of the averaging procedure as given below. With the pump polarization $\vec{e}_{\mathrm{pu}}$ along $\vec{e}_{1}=\vec{e}_{x}$ in a Cartesian coordinate system $\left(\vec{e}_{2}=\right.$ $\vec{e}_{y}$ and $\vec{e}_{3}=\vec{e}_{z}$ ) the six independent components are:

$$
\begin{aligned}
& \chi_{1111}^{(3) \mathrm{E}}=\frac{5}{35} \tilde{\gamma}_{0} L ; \quad \chi_{1221}^{(3) \mathrm{E}}=\frac{1}{35} \tilde{\gamma}_{0} L ; \\
& \chi_{1122}^{(3) \mathrm{E}}=\chi_{1212}^{(3) \mathrm{E}}=\frac{1}{35} \tilde{\gamma}_{0} L ; \\
& \chi_{2222}^{(3) \mathrm{E}}=\chi_{3333}^{(3) \mathrm{E}}=\frac{1}{35} \tilde{\gamma}_{0} L ; \\
& \chi_{2112}^{(3) \mathrm{E}}=\frac{1}{35} \tilde{\gamma}_{0} L ; \quad \chi_{2211}^{(3) \mathrm{E}}=\chi_{2121}^{(3) \mathrm{E}}=\frac{1}{35} \tilde{\gamma}_{0} L .
\end{aligned}
$$

The relative values are

$$
\chi_{1111}^{(3) E}: \chi_{2222}^{(3) E}: \chi_{1221}^{(3) E}: \chi_{1122}^{(3) E}: \chi_{2112}^{(3) E}: \chi_{2211}^{(3) E}=5: 1: 1: 1: 1: 1
$$

The depolarization ratio $\rho_{\mathrm{ANSS}}^{S_{0}}$ of the electronic ground state in the case of excitation induced anisotropy can be computed by combining Eqs. A1-A5:

$$
\rho_{\mathrm{ANIS}}^{S_{0}}=\frac{\chi_{2112}^{(3) \mathrm{E}}}{\chi_{1111}^{(3) \mathrm{E}}}=\frac{7-3 g_{1}}{21-15 g_{1}}
$$

For $g_{1}=0$ (i.e., no $S_{1}$ excited molecules) we find the isotropic case $\rho=1 / 3$ in agreement with Kleinman symmetry. In the limit of $g_{1}=1$, one obtains $\rho_{\text {ANIS }}=2 / 3$. In general, with some excited fraction $0<g_{1}<1$ the depolarization ratio has a value $1 / 3<\rho_{\text {ANIS }}<2 / 3$.

\section{APPENDIX B: DECOMPOSITION OF ELLIPTICALLY POLARIZED LIGHT INTO TWO LINEARLY POLARIZED WAVES}

In the following we adapt the analysis method of Ref. 5 . In the case of linearly polarized nonresonant signal (without pump) the intensity dependence on the analyzer angle $\epsilon$ would be

$$
I_{a}^{\mathrm{NR}}(\epsilon)=\left(P^{\mathrm{NR}}\right)^{2} \sin ^{2} \epsilon S(\epsilon)
$$

where sensitivity of the detection system to polarization direction is incorporated in the function $S(\epsilon)$.

$$
S(\epsilon)=\sin ^{2}\left(\epsilon-\phi^{\mathrm{NR}}\right)+k \cos ^{2}\left(\epsilon-\phi^{\mathrm{NR}}\right)
$$

with $\epsilon$ defined by the angle between the analyzer transmittance plane $\vec{e}_{A}$ and the normal to the nonresonant polarization $\vec{P}^{\mathrm{NR}}$, and $k$ the ratio of horizontal to vertical sensitivity of the detection system.

For the elliptically polarized CARS signal (with pump) the dependence can be written as

$$
I_{a}^{\mathrm{pu}}(\epsilon)=\left[a^{2} \sin ^{2}(\epsilon-\psi)+b^{2} \cos ^{2}(\epsilon-\psi)\right] S(\epsilon)
$$

where $a$ and $b$ are the semi-axes of the ellipse, and $\psi$ is the angle between the major semi-axis and the direction of the nonresonant polarization $\vec{P}^{\mathrm{NR}}$. The solid curves in Fig. 9 are fit results according to Eqs. B1 and B3 with the parameters: $P^{\mathrm{NR}}=(0.84 \pm 0.02), a=(0.99 \pm 0.03)$, $b=(0.08 \pm 0.07), k=(0.29 \pm 0.03)$ and $\psi=-(2.9 \pm$ $0.5)^{\circ}$.

The ellipse $I_{a}^{\text {pu }}(\epsilon)$ can be broken down into two linear polarized waves with amplitudes $P_{\xi}$ and $P_{\eta}$ and phase shift $\delta$ between them, where $P_{\xi}$ and $P_{\eta}$ are polarized parallel and perpendicular to the $P^{\mathrm{NR}}$-axis (see Fig. 8). $P_{\xi}$, $P_{\eta}$, and $\delta$ are calculated from:

$$
\begin{gathered}
P_{\xi}=\left(a^{2} \cos ^{2} \psi+b^{2} \sin ^{2} \psi\right)^{1 / 2}=0.922, \\
\vec{P}_{\xi}=P_{\xi} \vec{e}_{\xi} \quad \text { with } \vec{e}_{\xi} \| \vec{P}^{\mathrm{NR}} \\
P_{\eta}=\left(b^{2} \cos ^{2} \psi+a^{2} \sin ^{2} \psi\right)^{1 / 2}=0.086, \\
\quad \vec{P}_{\eta}=P_{\eta} e^{i \overrightarrow{e_{\eta}}} \quad \text { with } \vec{e}_{\eta} \perp \vec{P}^{\mathrm{NR}} \\
\delta=\arccos \left[\left(a^{2}-b^{2}\right) \cos \psi \sin \psi / P_{\xi} P_{\eta}\right]= \pm 125^{\circ} .
\end{gathered}
$$

As indicated before, these two polarizations are composed of the $\vec{P}^{\mathrm{NR}}$ and $\vec{P}^{\mathrm{E}}$ polarizations with phase difference $\theta^{\mathrm{E}}$.

$$
\begin{aligned}
& \vec{P}_{\xi}=\left(\left|\vec{P}^{\mathrm{NR}}\right|+\left|\vec{P}_{\xi}^{\mathrm{E}}\right| e^{i \theta^{\mathrm{E}}}\right) \vec{e}_{\xi} \\
& \vec{P}_{\eta}=\left( \pm\left|\vec{P}_{\eta}^{\mathrm{E}}\right| e^{i \theta^{\mathrm{E}}}\right) \vec{e}_{\eta^{\circ}} .
\end{aligned}
$$

Our task now is to find the amplitudes $\left|\vec{P}_{\xi}^{\mathrm{E}}\right|,\left|\vec{P}_{\eta}^{\mathrm{E}}\right|$ and the phase $\theta^{\mathrm{E}}$ from the sets in Eqs. B4-B6 and B7 and B8. From Eq. B7 it follows

$$
\left|\vec{P}_{\xi}\right|^{2}=\left(P^{\mathrm{NR}}\right)^{2}+2 P^{\mathrm{NR}} P_{\xi}^{\mathrm{E}} \cos \theta^{\mathrm{E}}+\left(P_{\xi}^{\mathrm{E}}\right)^{2} .
$$

From the definitions of the phases $\delta$ and $\theta^{\mathrm{E}}$ in Eqs. B5 and $\mathrm{B} 7$, one can find

$$
\arg \left(P_{\eta}\right)-\arg \left(P_{\xi}\right)=\delta
$$

and

$$
\arg \left(P_{\eta}\right)-\arg \left(P_{\xi}\right)=\theta^{\mathrm{E}}-\arctan \left\{\frac{P_{\xi}^{\mathrm{E}} \sin \theta^{\mathrm{E}}}{P^{\mathrm{NR}}+P_{\xi}^{\mathrm{E}} \cos \theta^{\mathrm{E}}}\right\}
$$

which gives

$$
P_{\xi}^{\mathrm{E}}=P^{\mathrm{NR}}\left(\frac{\tan \theta^{\mathrm{E}}-\tan \delta}{\tan \delta}\right) \cos \theta^{\mathrm{E}} .
$$

Substitution of B12 in B7 gives the phase shift $\theta^{\mathrm{E}}$

$$
\theta^{\mathrm{E}}=\arcsin \left( \pm \sin \delta \frac{P_{\xi}}{P^{\mathrm{NR}}}\right)
$$

and $P_{\xi}^{\mathrm{E}}$ is found by substituting Eq. B13 into Eq. B12. There are four possibilities for calculating $P_{\xi}^{\mathrm{E}}$ : (1) $\left(\theta_{+}^{\mathrm{E}}\right.$, $\left.\delta_{+}\right)$; (2) $\left(\theta_{+}^{\mathrm{E}}, \delta_{-}\right) ;(3)\left(\theta_{-}^{\mathrm{E}}, \delta_{+}\right)$; and (4) $\left(\theta_{-}^{\mathrm{E}}, \delta_{-}\right)$, but 1 and 4 (same phase) and 2 and 3 (opposite phase) give the same result. The two possible configurations of the vectors $P_{\xi}^{\mathrm{E}}$ and $P_{\eta \pm}^{\mathrm{E}}$, where $P_{\xi}^{\mathrm{E}}$ and $P_{\eta}^{\mathrm{E}}$ have opposite or the same phases, are represented in the complex plane in Fig. 10. The sum of $P^{\mathrm{NR}}$ and $P_{\xi}^{\mathrm{E}}$ gives the same $\left|P_{\eta}\right|$. The angle $\beta$ is given by

$$
\beta_{ \pm}=\arccos \left(\left(\left(P^{\mathrm{NR}}\right)^{2}+P_{\xi}^{2}-\left|\vec{P}_{\xi \pm}^{\mathrm{E}}\right|^{2}\right) / 2 P_{\xi} P^{\mathrm{NR}}\right) \text {. }
$$

With this method $\theta^{\mathrm{E}}$ is found to be $\theta^{\mathrm{E}}= \pm 74.8^{\circ}$. The two possible sets for $P_{\xi^{+}}^{\mathrm{E}}, P_{\eta^{+}}^{\mathrm{E}}, \beta_{+}$and $P_{\xi-}^{\mathrm{E}}, P_{\eta^{-}}^{\mathrm{E}}, \beta_{-}$, where $P_{\xi}^{\mathrm{E}}$ and $P_{\eta}^{\mathrm{E}}$ have opposite and the same phases, are given in the text (see the fourth part of the Results section). 